\title{
Epidemiology and Economic Burden of Cardiovascular Disease in Patients with Type 2 Diabetes Mellitus in Spain: A Systematic Review
}

\author{
Esther Artime · Irene Romera · Silvia Díaz-Cerezo · Elías Delgado
}

Received: February 17, 2021 / Accepted: April 12, 2021 / Published online: May 3, 2021

(C) The Author(s) 2021

\section{ABSTRACT}

Introduction: Cardiovascular disease (CVD) is a leading cause of morbidity and mortality in people with type 2 diabetes mellitus (T2DM). The objectives of this systematic literature review were to identify and synthesize published data describing the epidemiology and mortality of CVD in the T2DM population and the associated economic burden.

Methods: We conducted a systematic review searching the PubMed and MEDES databases

Supplementary Information The online version contains supplementary material available at https:// doi.org/10.1007/s13300-021-01060-8.

E. Artime $(\varangle) \cdot$ I. Romera $\cdot S$. Díaz-Cerezo

Eli Lilly and Company, Avda. de la Industria 30, Alcobendas, 28108 Madrid, Spain

e-mail: artime_esther@lilly.com

E. Delgado

Department of Endocrinology and Nutrition,

University of Oviedo, Oviedo, Spain

E. Delgado

Central University Hospital of Asturias, Oviedo, Spain

E. Delgado

Health Research Institute of the Principality of

Asturias (ISPA), Oviedo, Spain

E. Delgado

Spanish Biomedical Research Network in Rare

Diseases, Madrid, Spain from 2009 to 2019 using predefined selection criteria. Peer-reviewed observational studies reporting primary or secondary data on CVD prevalence, incidence, mortality, resource use and costs in patients with T2DM in Spain, written in English and Spanish, were included. Data were tabulated and summarized descriptively.

Results: Of 706 articles identified, 52 were included in the review. Most studies were based on data from hospital discharge databases and registries. The reported prevalence of CVD among patients with T2DM ranged from 6.9 to $40.8 \%$. The prevalence of coronary heart disease ranged from 4.7 to $37 \%$, stroke from 3.5 to $19.6 \%$, peripheral artery disease from 2.5 to $13.0 \%$, and heart failure from 4.3 to $20.1 \%$. Inhospital CVD mortality rates ranged from 5.6 to $10.8 \%$. Direct costs due to CVD in hospitalized patients with T2DM were increased (>50\%) compared with patients without CVD. No studies analysed indirect costs of CVD in patients with T2DM.

Conclusions: The burden of CVD among patients with T2DM, combined with the elevated costs of care, highlights the importance of early prevention as part of integrated management of the disease to improve clinical and economic outcomes.

Keywords: Burden of disease; Cardiovascular disease; Costs; Diabetes mellitus, type 2; Incidence; Mortality; Prevalence 


\section{Key Summary Points}

About one-third of patients with type 2 diabetes mellitus (T2DM) have cardiovascular disease (CVD), which is the leading cause of mortality in this group.

Total direct costs associated with CVD complications in patients with T2DM can be twice those of patients without CVD.

The literature on CVD complications in the T2DM population is methodologically diverse, which limits comparative analysis and pooling of data.

In studies published between 2009 and 2019, the prevalence of CVD among patients with T2DM in Spain ranged from 7 to $41 \%$, and in-hospital mortality rates due to CVD were between 6 and 11\%.

Given the high burden of treating CVD complications in patients with T2DM, early prevention of CVD is critical and should be a priority in these patients.

\section{DIGITAL FEATURES}

This article is published with digital features, including a summary slide, to facilitate understanding of the article. To view digital features for this article go to https://doi.org/10.6084/ m9.figshare.14356655.

\section{INTRODUCTION}

Cardiovascular disease (CVD) morbidity affects approximately $32 \%$ of patients with type 2 diabetes mellitus (T2DM) worldwide and is a leading cause of mortality [1]. According to the International Diabetes Federation, people with diabetes are two- to threefold more likely to develop CVD than their non-diabetic counterparts [2].
Generally, the cardiovascular (CV)-related effects of hyperglycaemia are separated into macrovascular complications (coronary heart disease [CHD], stroke and peripheral arterial disease $[\mathrm{PAD}]$ ) and microvascular complications (diabetic nephropathy, neuropathy and retinopathy). The central pathological mechanism in macrovascular disease is atherosclerosis, but the precise mechanisms through which hyperglycaemia promotes atherosclerotic plaque formation are not fully understood [3].

Since T2DM complications are mostly CV in nature, the control of $\mathrm{CV}$ risk factors should be an integral part of the treatment of T2DM [4-6]. In addition to glycaemic control, risk factors for CVD in patients with T2DM include hypertension, dyslipidaemia, obesity, lack of physical activity and smoking [7]. Of these, blood pressure and lipid control have been regarded as the two most important risk factors for CVD in recent guidelines $[8,9]$. The 2020 American Diabetes Association (ADA) guidelines indicate that CVD risk factors should be assessed annually in all patients with T2DM and that their control is essential for the optimal management of T2DM [8].

In recent years, focus has increasingly been on the management of CV risks in the context of diabetic pharmacotherapy. There is strong interest in demonstrating both the CV safety and the $\mathrm{CV}$ benefit of all antidiabetic agents, especially for macrovascular complications. $\mathrm{CV}$ outcomes trials have evaluated the CV safety and efficacy profile of some of these agents [10]. As a result, the recently published ADA/European Association for the Study of Diabetes (EASD) consensus statement recommends the use of sodium-glucose cotransporter-2 inhibitors (SGLT2i) and glucagon-like peptide-1 receptor agonists (GLP-1 RAs) in patients with T2DM with established CVD or high CV risk $[5,6]$. Some individual agents within these drug classes have been shown to have CV benefits for risk reduction of $\mathrm{CV}$ events in patients with T2DM [11].

CVDs have been recognized as having a major impact on the direct costs of treating patients with T2DM. A recent review on the global economic burden of treating CV comorbidities in patients with T2DM found that direct 
medical costs were significantly increased in this population [12]. This study found that the median increase in costs due to treating patients with CVD complications was $112 \%$ (range 47-196\%) compared with patients with T2DM alone [12]. However, this review also highlighted that the number of studies devoted to this issue was generally limited and that more research should be conducted to inform health economic evaluations.

A recent worldwide systematic literature review found that, based on seven articles published between 2009 and 2016, the prevalence of CVD associated with T2DM in Spain was $29.8 \%$ [1]. However, the worldwide scope of this systematic review precluded inclusion of other studies from Spain and a more detailed analysis of the data derived from Spanish patients. It also did not include incidence or associated costs. As the different CVDs are associated with distinct risks in patients with T2DM [13, 14], having precise and current epidemiological information is essential in resource allocation and therapy evaluation. The purpose of this systematic review was to gather and synthesize the studies published in the last 10 years reporting data on the prevalence and incidence of CVD, CVD-related mortality and associated burdens and costs in adult patients with T2DM in Spain.

\section{METHODS}

The systematic search and procedures were performed following the Preferred Reporting Items for Systematic Reviews and Meta-Analyses (PRISMA) guidelines. The objective of this systematic literature review was to identify and synthesize the data available on the overall prevalence of CVD in adults with T2DM in Spain; the incidence of CVD in adults with T2DM and CV risk factors in Spain; the incidence of a subsequent CVD event in adults with established CVD and T2DM in Spain; the mortality due to CVD in patients with T2DM in Spain; health resource utilization (HRU) and costs associated with $\mathrm{CV}$ events in adults with T2DM in Spain (direct and indirect costs, total costs and information about impact on work); and the costs associated with $\mathrm{CV}$ events in patients with T2DM with or without established CVD.

This article is based on previously conducted studies and does not contain any new studies with human participants or animals performed by any of the authors.

\section{Selection Criteria}

Selection criteria focused on peer-reviewed articles with epidemiological and health economics data published between January 2009 and December 2019. Observational studies (cross-sectional, cohort studies) reporting primary (e.g. surveys) or secondary (e.g., databases) data on CVD prevalence, incidence, mortality, costs or resource use in adults (aged $\geq 18$ years) with T2DM were eligible. Included articles contained data on macrovascular CVD (e.g. CVD overall, CHD, haemorrhagic or ischaemic cerebrovascular disease, PAD, heart failure $[\mathrm{HF}]$, atherosclerosis). Studies in which data on patients with T2DM were present but not the main focus of the study were included only if the diabetic population was fully characterized (e.g. studies on population-level rates of CVD or conditions). All publications reviewed were in English or Spanish.

We excluded narrative and systematic reviews, clinical trials, letters, opinion articles and editorials. Abstracts, conference proceedings and articles on economic models were not considered, and studies focused on diabetic retinopathy and other microvascular complications were excluded.

\section{Literature Search}

The search was undertaken between 15 November and 7 December 2019. The primary electronic databases searched were MEDLINE (PubMed) and MEDES (Spanish bibliographic database in health sciences). The search terms for MEDLINE used the following medical subject headings and standard keywords in all fields and in various combinations: cardiovascular disease; myocardial; cardiac; cardiovascular; coronary; stroke; cerebrovascular; angina; diabetes mellitus, type 2 ; diabetes complications; 
prevalence; incidence; epidemiology; morbidity; burden; costs and cost analysis; economic impact; resources; and Spain. Search terms for MEDES used the equivalent keywords in Spanish in various combinations: diabetes tipo 2; cardiovascular; cardiaco; infarto; ictus; prevalencia; incidencia; epidemiología; carga; costes. The literature found was complemented with articles identified through manual searches of the bibliographies of included studies.

\section{Data Extraction and Analysis}

Two reviewers independently screened and selected articles, and a third reviewer arbitrated in case of any doubt on eligibility. Data from each article were extracted and subsequently verified by an independent reviewer.

From each selected article, the following data were extracted and tabulated: data collection period; geographic scope (i.e. national, regional, local); study design; data source; number of patients with T2DM included; outcome of interest reported (i.e. prevalence, incidence, mortality, costs); and type of event (i.e. CVD overall, CHD, cerebrovascular accident [CVA], PAD, HF). The definition of CVD used in each study was also retrieved.

Studies with prevalence data were classified according to the study population: general population with diabetes mellitus versus special populations, such as those with a predetermined age range (e.g. elderly) or with additional comorbidities (e.g. kidney disease). The following patient characteristics were extracted: sex (\% male), age (mean, years), disease duration (mean, years) and glycated haemoglobin (HbA1c; \%). Data on prevalence, reported as the number of patients with the event of interest present in the study population, were extracted and presented by event type (CVD overall, CHD, CVA, PAD, HF). Studies with a national versus a regional scope were presented separately. The prevalence of CVD overall by geography was presented graphically.

Incidence data (new occurrences of the event of interest over a period of time) were extracted as reported in the selected articles (e.g. rate per 100,000 inhabitants, age-adjusted, percentage, rate per 1000 person-years). Recurrences of events in patients with established CVD are described separately. Incidence and mortality data are presented by event type (CVD overall, CHD and myocardial infarction, CVA, aortic aneurism and dissection, acute lower limb ischaemia, PAD, HF) and by geographical scope (national, regional). The length of the follow-up period was obtained for incidence and mortality outcomes. Costs were extracted as mean cost per episode per patient in some studies, whereas others reported total cost per patient-year. Where available, the cost components (e.g. hospitalizations, pharmacological treatment) were obtained. The year for cost calculation is also presented.

No formal statistical analysis was performed. Data are reported as ranges from the various studies. Given the heterogeneity of the data, no meta-analysis or overall quantitative synthesis was undertaken.

\section{RESULTS}

The article selection process is shown in Fig. 1. The initial search identified 114 papers of possible interest, and 52 met the selection criteria after systematic and detailed examination of the full article. Table 1 lists these studies along with their methodological characteristics and main outcomes. In total, 22 studies had a crosssectional design, 20 were retrospective and ten were prospective cohort studies. Electronic Supplementary Material (ESM) Table S1 provides detailed descriptions of the definitions of CVD in each article and shows that most studies used codes from the International Statistical Classification of Diseases and Related Health Problems, Ninth or Tenth Revision (ICD-9 or ICD-10) to identify the presence of CVD or the occurrence of $\mathrm{CV}$ events.

Regarding epidemiological parameters, this review included 31 articles published between 2009 and 2019 that analysed the prevalence of CVD overall or a type of CVD in patients with T2DM in Spain $[15,16,19,20,23-25$, $28-35,37,42,43,45,52,54-57,59-63,65,66]$, 20 articles that analysed incidence $[15,21-23,27,36,38-41,44,46,48-51,53$, 


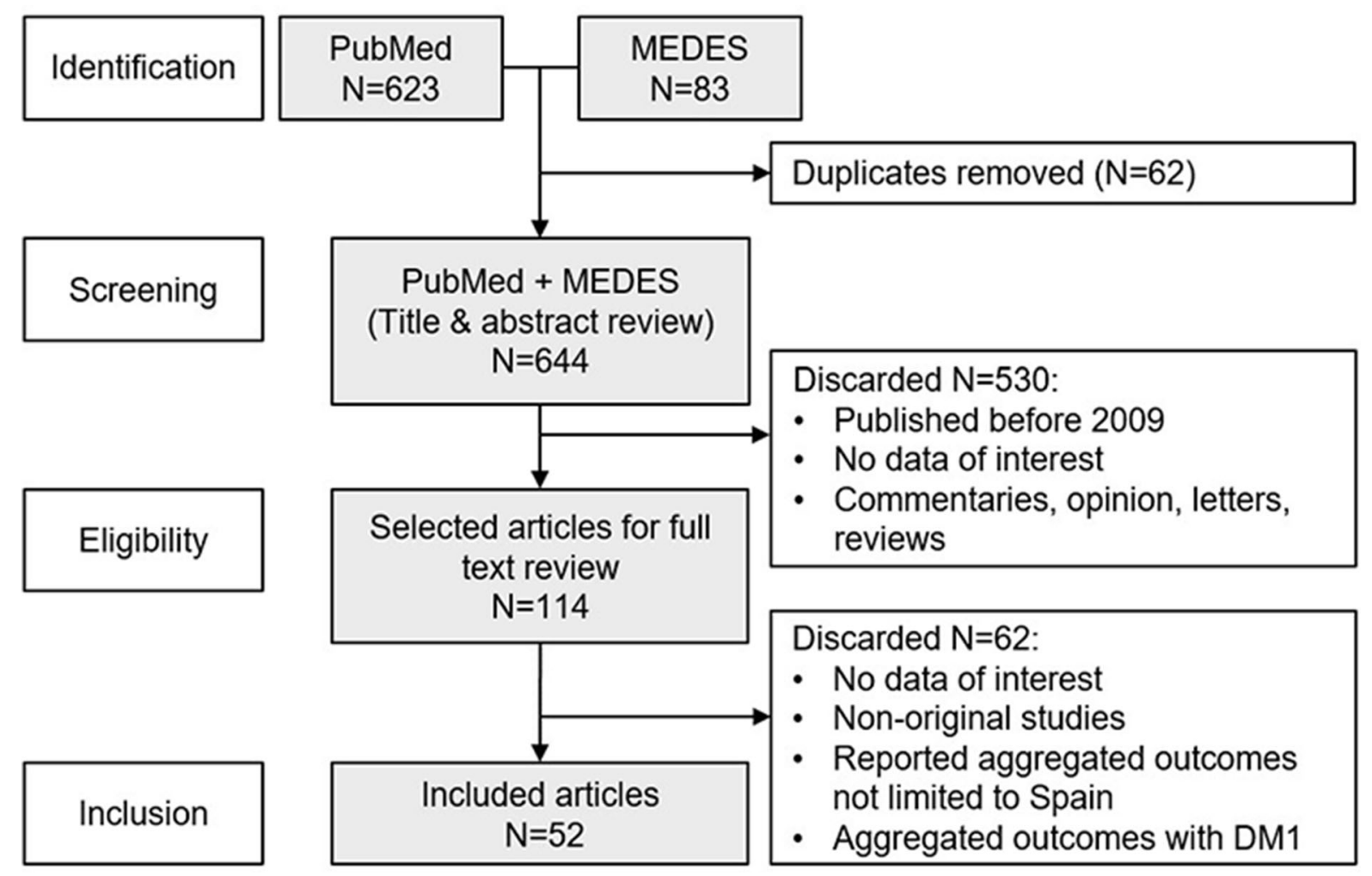

Fig. 1 Flow diagram showing the selection process of the included articles. DMI Type 1 diabetes mellitus

$56,58,64]$, and 18 articles that analysed mortality $[16,18,21,22,25,27,30,31,38-41,44$, $46,56,57,60,66]$.

Of the articles reporting epidemiology data, 20 included countrywide (national) data, 17 were regional (autonomous communities) and 11 were local (specific hospitals, cities, or districts). Catalonia was the region with the most studies (18 articles), followed by Madrid (five), Valencia (four), the Basque Country (two) and Asturias (one).

Five studies reported on HRU and/or costs associated with CVD in patients with T2DM $[17,26,37,40,62]$.

\section{Prevalence of CVD in Patients with T2DM}

Table 2 summarizes the prevalence or frequency of CV comorbidities in the samples of patients with T2DM described in the articles. Seven studies analysed the overall prevalence of CVD at the national level $[28,34,52,54,55,59,66]$. A recent countrywide study analysed the prevalence of CVD in 619,188 patients with T2DM hospitalized in Spain in 2015 and is the largest study to date on this topic [66].

The regional studies included in this systematic review analysed evaluated patients from Catalonia (nine studies), the Basque Country (two studies), Madrid (two studies) and Valencia (one study). Of these, the studies including the largest number of patients with T2DM were those from Catalonia (373,185 patients) [42], Valencia $(350,015$ patients) [62], the Basque Country (149,015 patients) [15] and Madrid (3407 patients) [19].

Considering studies of the general population with T2DM, the overall prevalence/frequency of CVD ranged from 6.9 to $40.8 \%$ in the 14 studies reporting this value (Table 2; Fig. 2). In these studies, the range of prevalence was $17.0-40.8 \%$ at the national level and $6.9-30.4 \%$ at the regional level.

Most studies detailed the type of CVD (Table 2). For the general diabetic population, prevalence was $4.7-37.0 \%$ for CHD (17 studies) 
Table 1 Articles included in this systematic review on the epidemiology of cardiovascular disease and health resource utilization and costs associated with cardiovascular events in adults with type 2 diabetes mellitus in Spain (2009-2019)

\begin{tabular}{|c|c|c|c|c|c|c|}
\hline $\begin{array}{l}\text { First author } \\
\text { of article } \\
\text { [citation } \\
\text { number] }\end{array}$ & $\begin{array}{l}\text { Data } \\
\text { collection } \\
\text { period }\end{array}$ & $\begin{array}{l}\text { Geographic } \\
\text { range }\end{array}$ & Study design & Data source & $\begin{array}{l}\text { Patients } \\
\text { with } \\
\text { T2DM } \\
(N)\end{array}$ & $\begin{array}{l}\text { CV-related } \\
\text { outcomes }\end{array}$ \\
\hline $\begin{array}{l}\text { Alonso-Morán } \\
\text { [15] }\end{array}$ & $2007-2011$ & $\begin{array}{l}\text { Regional: } \\
\text { Basque } \\
\text { Country }\end{array}$ & $\begin{array}{l}\text { Retrospective } \\
\text { cohort }\end{array}$ & $\begin{array}{l}\text { Public Health } \\
\text { Service databases }\end{array}$ & 149,015 & $\begin{array}{c}\text { Prevalence (CHD, } \\
\text { CVA, PAD, HF); } \\
\text { incidence (ACS) }\end{array}$ \\
\hline Ares [16] & $1998-2016$ & $\begin{array}{l}\text { Regional: } \\
\text { Asturias }\end{array}$ & $\begin{array}{l}\text { Population- } \\
\text { based, } \\
\text { retrospective } \\
\text { cohort }\end{array}$ & $\begin{array}{l}\text { Random sampling } \\
\text { from the general } \\
\text { population }\end{array}$ & 48 & Mortality \\
\hline Arrieta $[17]$ & 2012 & $\begin{array}{l}\text { Regional: } \\
\text { Madrid } \\
\text { Community }\end{array}$ & $\begin{array}{r}\text { Cross-sectional } \\
\text { cohort study }\end{array}$ & $\begin{array}{l}\text { Interviews with } \\
\text { primary care } \\
\text { physicians, expert } \\
\text { panel analysis }\end{array}$ & 3268 & Costs (CVD) \\
\hline Arrieta $[18]$ & $2007-2010$ & $\begin{array}{l}\text { Regional: } \\
\text { Madrid } \\
\text { Community }\end{array}$ & $\begin{array}{r}\text { Cross-sectional } \\
\text { cohort study }\end{array}$ & $\begin{array}{l}\text { Interviews with } \\
\text { primary care } \\
\text { physicians, expert } \\
\text { panel analysis }\end{array}$ & 3268 & $\begin{array}{c}\text { Incidence (CHD, } \\
\text { CVA, PAD) }\end{array}$ \\
\hline Arrieta [19] & 2007 & $\begin{array}{l}\text { Regional: } \\
\text { Madrid } \\
\text { Community }\end{array}$ & Cross-sectional & $\begin{array}{l}\text { Random sampling in } \\
\text { primary healthcare } \\
\text { centres }\end{array}$ & 3268 & $\begin{array}{l}\text { Prevalence (CHD, } \\
\text { CVA, PAD, HF) }\end{array}$ \\
\hline $\begin{array}{l}\text { Barrot-de la } \\
\text { Puente [20] }\end{array}$ & 2011 & $\begin{array}{l}\text { Regional: } \\
\text { Catalonia }\end{array}$ & $\begin{array}{l}\text { Population- } \\
\text { based, cross- } \\
\text { sectional }\end{array}$ & Electronic database & 318,020 & $\begin{array}{l}\text { Prevalence (CVD, } \\
\text { CHD, CVA, } \\
\text { PAD, HF) }\end{array}$ \\
\hline Cano $[21]$ & $1993-2008$ & $\begin{array}{l}\text { Regional: } \\
\text { Catalonia }\end{array}$ & $\begin{array}{l}\text { Population- } \\
\text { based } \\
\text { prospective } \\
\text { cohort }\end{array}$ & $\begin{array}{l}\text { Recruitment in } \\
\text { primary care } \\
\text { centres }\end{array}$ & 2260 & $\begin{array}{l}\text { Incidence (ACS, } \\
\text { CHD); mortality }\end{array}$ \\
\hline Camafort [22] & 2003-2009 & National & $\begin{array}{l}\text { Prospective } \\
\text { cohort }\end{array}$ & $\begin{array}{l}\text { Hospital-based Risk } \\
\text { Factors in Arterial } \\
\text { Disease (FRENA) } \\
\text { registry }\end{array}$ & 974 & $\begin{array}{l}\text { Incidence (CHD, } \\
\text { CVA, PAD, } \\
\text { AMI); mortality }\end{array}$ \\
\hline $\begin{array}{l}\text { Carrasco- } \\
\text { Sánchez [23] }\end{array}$ & $2008-2011$ & National & $\begin{array}{l}\text { Prospective } \\
\text { cohort }\end{array}$ & $\begin{array}{l}\text { Spanish National } \\
\text { Registry on Heart } \\
\text { Failure (RICA) }\end{array}$ & 490 & $\begin{array}{l}\text { Prevalence (CHD, } \\
\text { CVA, PAD, HF) }\end{array}$ \\
\hline Catalán [24] & $2012-2013$ & $\begin{array}{l}\text { Local: } \\
\text { Barcelona }\end{array}$ & Cross-sectional & $\begin{array}{l}\text { Primary care centres } \\
\text { survey of newly } \\
\text { diagnosed T2DM } \\
\text { patients }\end{array}$ & 106 & $\begin{array}{l}\text { Prevalence } \\
\quad \text { (atherosclerosis) }\end{array}$ \\
\hline
\end{tabular}


Table 1 continued

\begin{tabular}{|c|c|c|c|c|c|c|}
\hline $\begin{array}{l}\text { First author } \\
\text { of article } \\
\text { [citation } \\
\text { number] }\end{array}$ & $\begin{array}{l}\text { Data } \\
\text { collection } \\
\text { period }\end{array}$ & $\begin{array}{l}\text { Geographic } \\
\text { range }\end{array}$ & Study design & Data source & $\begin{array}{l}\text { Patients } \\
\text { with } \\
\text { T2DM } \\
(N)\end{array}$ & $\begin{array}{l}\text { CV-related } \\
\text { outcomes }\end{array}$ \\
\hline $\begin{array}{l}\text { Clua-Espuny } \\
{[25]}\end{array}$ & $2013-2016$ & $\begin{array}{l}\text { Local: Terres de } \\
\text { l'Ebre health } \\
\text { area } \\
\text { (Catalonia) }\end{array}$ & $\begin{array}{l}\text { Retrospective } \\
\text { cohort study }\end{array}$ & $\begin{array}{l}\text { Catalonian Health } \\
\text { Institute Registry } \\
\text { of complex } \\
\text { chronic patients }\end{array}$ & 494 & $\begin{array}{l}\text { Prevalence (CHD, } \\
\text { CVA, PAD, HF); } \\
\text { mortality }\end{array}$ \\
\hline Crespo [26] & 2012 & National & Retrospective & Various databases & 544,515 & $\begin{array}{l}\text { Costs (CVD, PAD, } \\
\text { CVA) }\end{array}$ \\
\hline $\begin{array}{l}\text { De Miguel- } \\
\text { Yanes [27] }\end{array}$ & 2002-2014 & National & $\begin{array}{l}\text { Retrospective } \\
\text { study }\end{array}$ & $\begin{array}{l}\text { National Hospital } \\
\text { Discharge } \\
\text { Database }\end{array}$ & 130,011 & $\begin{array}{l}\text { Incidence (AAD, } \\
\text { ALLI, AMI, CVA, } \\
\text { CVD); mortality }\end{array}$ \\
\hline De Pablos [28] & 2007 & National & Cross-sectional & $\begin{array}{l}\text { Primary care centres } \\
\text { survey }\end{array}$ & 601 & $\begin{array}{l}\text { Prevalence (CHD, } \\
\text { CVA, PAD) }\end{array}$ \\
\hline Ferrer [29] & 2009 & $\begin{array}{l}\text { Local: Baix } \\
\text { Llobregat } \\
\text { (Catalonia) }\end{array}$ & $\begin{array}{l}\text { Population- } \\
\text { based, } \\
\text { prospective } \\
\text { cohort }\end{array}$ & $\begin{array}{l}\text { Random sampling in } \\
\text { primary health } \\
\text { centres }\end{array}$ & 85 & $\begin{array}{l}\text { Prevalence (CHD, } \\
\text { CVA, PAD, HF) }\end{array}$ \\
\hline $\begin{array}{l}\text { Flores-Le Roux } \\
\quad[30]\end{array}$ & $2000-2002$ & $\begin{array}{l}\text { Local: Hospital } \\
\text { del Mar } \\
\text { (Catalonia) }\end{array}$ & $\begin{array}{l}\text { Retrospective } \\
\text { cohort }\end{array}$ & $\begin{array}{l}\text { Patients admitted to } \\
\text { hospital CV unit }\end{array}$ & 149 & $\begin{array}{l}\text { Prevalence (CHD, } \\
\text { CVA, PAD); } \\
\text { mortality }\end{array}$ \\
\hline Formiga [31] & 2013-2014 & $\begin{array}{l}\text { Local: Bellvitge } \\
\text { University } \\
\text { Hospital } \\
\text { (Catalonia) }\end{array}$ & $\begin{array}{l}\text { Retrospective } \\
\text { cohort }\end{array}$ & $\begin{array}{l}\text { Hospital admissions } \\
\text { data }\end{array}$ & 240 & $\begin{array}{l}\text { Prevalence (CHD, } \\
\text { CVA); mortality }\end{array}$ \\
\hline $\begin{array}{l}\text { Franch-Nadal } \\
\quad[32]\end{array}$ & $\begin{array}{l}\text { Up to } \\
2009\end{array}$ & $\begin{array}{l}\text { Regional: } \\
\text { Catalonia }\end{array}$ & $\begin{array}{l}\text { Population- } \\
\text { based, cross- } \\
\text { sectional }\end{array}$ & $\begin{array}{l}\text { Database of } \\
\text { electronic medical } \\
\text { records }\end{array}$ & 286,791 & Prevalence (CVD) \\
\hline Gámez [33] & $2011-2012$ & National & Cross-sectional & $\begin{array}{l}\text { Cardiology } \\
\text { consultation } \\
\text { sampling } \\
\text { (SIRENA study) }\end{array}$ & 257 & $\begin{array}{l}\text { Prevalence (HF, } \\
\text { stroke, PAD) in } \\
\text { women with } \\
\text { CHD }\end{array}$ \\
\hline $\begin{array}{l}\text { González- } \\
\text { Juanatey } \\
{[34]}\end{array}$ & - & National & Cross-sectional & $\begin{array}{c}\text { DIVA registry } \\
\text { subanalysis }\end{array}$ & 2444 & $\begin{array}{l}\text { Prevalence (CHD, } \\
\text { CVA, PAD) }\end{array}$ \\
\hline Gual [35] & - & National & $\begin{array}{l}\text { Prospective } \\
\text { cohort }\end{array}$ & $\begin{array}{l}\text { LONGEVO-SCA } \\
\text { registry of ACS } \\
\text { patients } \\
\text { aged } \geq 80 \text { years }\end{array}$ & 212 & $\begin{array}{l}\text { Prevalence (CHD, } \\
\text { CVA, PAD) in } \\
\text { ACS patients }\end{array}$ \\
\hline
\end{tabular}


Table 1 continued

\begin{tabular}{|c|c|c|c|c|c|c|}
\hline $\begin{array}{l}\text { First author } \\
\text { of article } \\
\text { [citation } \\
\text { number] }\end{array}$ & $\begin{array}{l}\text { Data } \\
\text { collection } \\
\text { period }\end{array}$ & $\begin{array}{l}\text { Geographic } \\
\text { range }\end{array}$ & Study design & Data source & $\begin{array}{l}\text { Patients } \\
\text { with } \\
\text { T2DM } \\
(N)\end{array}$ & $\begin{array}{l}\text { CV-related } \\
\text { outcomes }\end{array}$ \\
\hline $\begin{array}{l}\text { Jiménez- } \\
\text { Trujillo [36] }\end{array}$ & 2001-2012 & National & $\begin{array}{l}\text { Retrospective } \\
\text { cohort }\end{array}$ & $\begin{array}{l}\text { National hospital } \\
\text { discharge database }\end{array}$ & 10,491 & $\begin{array}{l}\text { Incidence (thoracic } \\
\text { aortic aneurysm) }\end{array}$ \\
\hline Jurado [37] & 2001-2002 & $\begin{array}{l}\text { Local: Girona } \\
\text { (Catalonia) }\end{array}$ & Cross-sectional & $\begin{array}{l}\text { Random sampling in } \\
\text { primary care } \\
\text { centres }\end{array}$ & 307 & $\begin{array}{c}\text { Prevalence (CVD, } \\
\text { CHD, PAD) }\end{array}$ \\
\hline $\begin{array}{l}\text { López-de- } \\
\text { Andrés [38] }\end{array}$ & 2003-2012 & National & $\begin{array}{l}\text { Retrospective } \\
\text { cohort }\end{array}$ & $\begin{array}{l}\text { Spanish National } \\
\text { Hospital Database }\end{array}$ & 19,232 & $\begin{array}{c}\text { Incidence (AAA); } \\
\text { mortality (AAA) }\end{array}$ \\
\hline $\begin{array}{l}\text { López-de- } \\
\text { Andrés [39] }\end{array}$ & 2001-2010 & National & $\begin{array}{l}\text { Retrospective } \\
\text { cohort }\end{array}$ & $\begin{array}{l}\text { Spanish National } \\
\text { Hospital Database }\end{array}$ & 155,676 & $\begin{array}{l}\text { Incidence (AMI); } \\
\text { mortality (AMI) }\end{array}$ \\
\hline $\begin{array}{l}\text { López-de- } \\
\text { Andrés [40] }\end{array}$ & 2001-2006 & National & $\begin{array}{l}\text { Retrospective } \\
\text { cohort }\end{array}$ & $\begin{array}{l}\text { Spanish National } \\
\text { Hospital Database }\end{array}$ & 90,835 & $\begin{array}{l}\text { Incidence (AMI); } \\
\text { mortality (AMI); } \\
\text { costs (AMI) }\end{array}$ \\
\hline $\begin{array}{l}\text { Navarro-Pérez } \\
\text { [41] }\end{array}$ & $2008-2012$ & $\begin{array}{l}\text { Regional: } \\
\text { Valencia } \\
\text { Community }\end{array}$ & $\begin{array}{l}\text { Population- } \\
\text { based, } \\
\text { prospective } \\
\text { cohort }\end{array}$ & $\begin{array}{l}\text { Electronic health } \\
\text { records }\end{array}$ & 19,140 & $\begin{array}{c}\text { Incidence (CHD, } \\
\text { stroke) by HbAlc } \\
\text { level; mortality }\end{array}$ \\
\hline $\begin{array}{l}\text { Mata-Cases } \\
\text { [42] }\end{array}$ & 2010-2016 & $\begin{array}{l}\text { Regional: } \\
\text { Catalonia }\end{array}$ & $\begin{array}{l}\text { Population- } \\
\text { based, cross- } \\
\text { sectional }\end{array}$ & $\begin{array}{l}\text { Registry of primary } \\
\text { health care system }\end{array}$ & 373,185 & $\begin{array}{l}\text { Prevalence (CVD, } \\
\text { HF) }\end{array}$ \\
\hline $\begin{array}{l}\text { Mata-Cases } \\
{[43]}\end{array}$ & 2011 & $\begin{array}{l}\text { Regional: } \\
\text { Catalonia }\end{array}$ & $\begin{array}{l}\text { Population- } \\
\text { based, cross- } \\
\text { sectional }\end{array}$ & $\begin{array}{l}\text { Registry of primary } \\
\text { health care system } \\
\text { and SIDIAP } \\
\text { database }\end{array}$ & 126,811 & $\begin{array}{l}\text { Prevalence (CHD, } \\
\text { CVA, PAD, HF); } \\
\text { Costs (CVD, HF) }\end{array}$ \\
\hline $\begin{array}{l}\text { Mata-Cases } \\
{[44]}\end{array}$ & 1991-2016 & $\begin{array}{l}\text { Local: Sant } \\
\text { Adrià de } \\
\text { Besòs } \\
\text { (Catalonia) }\end{array}$ & $\begin{array}{l}\text { Retrospective } \\
\text { cohort }\end{array}$ & $\begin{array}{l}\text { Primary health } \\
\text { centre }\end{array}$ & 469 & $\begin{array}{l}\text { Incidence (CVD, } \\
\text { AMI, CVA, } \\
\text { PAD); mortality }\end{array}$ \\
\hline $\begin{array}{l}\text { Mata-Cases } \\
{[45]}\end{array}$ & $1991-2000$ & $\begin{array}{l}\text { Local: Sant } \\
\text { Adrià de } \\
\text { Besòs } \\
\text { (Catalonia) }\end{array}$ & $\begin{array}{l}\text { Retrospective } \\
\text { cohort }\end{array}$ & $\begin{array}{l}\text { Registry in single } \\
\text { primary health } \\
\text { centre }\end{array}$ & 487 & $\begin{array}{l}\text { Prevalence (CVD, } \\
\text { CHD, CVA, } \\
\text { PAD) }\end{array}$ \\
\hline Mostaza [46] & - & National & $\begin{array}{l}\text { Prospective } \\
\text { cohort }\end{array}$ & $\begin{array}{l}\text { Registry in major } \\
\text { hospitals }\end{array}$ & 498 & $\begin{array}{l}\text { Incidence (CVD, } \\
\text { AMI); mortality }\end{array}$ \\
\hline Mundet [47] & 2003 & $\begin{array}{l}\text { Regional: } \\
\text { Catalonia }\end{array}$ & Cross-sectional & $\begin{array}{l}\text { Primary care health } \\
\text { centres }\end{array}$ & 4298 & $\begin{array}{l}\text { Prevalence (CVD, } \\
\text { CHD, CVA) }\end{array}$ \\
\hline
\end{tabular}


Table 1 continued

\begin{tabular}{|c|c|c|c|c|c|c|}
\hline $\begin{array}{l}\text { First author } \\
\text { of article } \\
\text { [citation } \\
\text { number] }\end{array}$ & $\begin{array}{l}\text { Data } \\
\text { collection } \\
\text { period }\end{array}$ & $\begin{array}{l}\text { Geographic } \\
\text { range }\end{array}$ & Study design & Data source & $\begin{array}{l}\text { Patients } \\
\text { with } \\
\text { T2DM } \\
(N)\end{array}$ & $\begin{array}{l}\text { CV-related } \\
\text { outcomes }\end{array}$ \\
\hline $\begin{array}{l}\text { Muñoz-Rivas } \\
{[48]}\end{array}$ & $2001-2015$ & National & $\begin{array}{l}\text { Retrospective } \\
\text { cohort }\end{array}$ & $\begin{array}{l}\text { Spanish National } \\
\text { Hospital } \\
\text { Discharge } \\
\text { Database }\end{array}$ & 553,775 & $\begin{array}{l}\text { Incidence (HF); } \\
\text { mortality (HF) }\end{array}$ \\
\hline $\begin{array}{l}\text { Muñoz-Rivas } \\
\text { [49] }\end{array}$ & $2003-2012$ & National & $\begin{array}{l}\text { Retrospective } \\
\text { cohort }\end{array}$ & $\begin{array}{l}\text { Spanish National } \\
\text { Hospital } \\
\text { Discharge } \\
\text { Database }\end{array}$ & 33,230 & $\begin{array}{l}\text { Incidence } \\
\text { (haemorrhagic } \\
\text { stroke) }\end{array}$ \\
\hline $\begin{array}{l}\text { Muñoz-Rivas } \\
\text { [50] }\end{array}$ & $2003-2012$ & National & $\begin{array}{l}\text { Retrospective } \\
\text { cohort }\end{array}$ & $\begin{array}{l}\text { Spanish National } \\
\text { Hospital } \\
\text { Discharge } \\
\text { Database }\end{array}$ & 130,858 & $\begin{array}{l}\text { Incidence (ischaemic } \\
\text { stroke) }\end{array}$ \\
\hline $\begin{array}{l}\text { Orozco- } \\
\text { Beltrán [51] }\end{array}$ & $2008-2012$ & $\begin{array}{l}\text { Regional: } \\
\text { Valencia } \\
\text { Community }\end{array}$ & $\begin{array}{l}\text { Prospective } \\
\text { cohort }\end{array}$ & Registry & 19,144 & $\begin{array}{l}\text { Incidence (CHD, } \\
\text { CVA) }\end{array}$ \\
\hline Ortega [52] & 2009-2010 & National & $\begin{array}{l}\text { Population- } \\
\text { based, cross- } \\
\text { sectional }\end{array}$ & $\begin{array}{l}\text { Nurse-administered } \\
\text { questionnaire and } \\
\text { physician reports }\end{array}$ & 312 & $\begin{array}{l}\text { Prevalence (CVD, } \\
\text { CHD, CVA, } \\
\text { PAD) }\end{array}$ \\
\hline Palanca [53] & $2010-2012$ & National & $\begin{array}{l}\text { Prospective } \\
\text { cohort }\end{array}$ & $\begin{array}{l}\text { Database from the } \\
\text { NEFRONA study }\end{array}$ & 698 & $\begin{array}{l}\text { Incidence (CVD) in } \\
\mathrm{CKD} \text { patients }\end{array}$ \\
\hline $\begin{array}{l}\text { Peña- } \\
\text { Longobardo } \\
{[54]}\end{array}$ & $2011-2012$ & National & Cross-sectional & $\begin{array}{c}\text { Spanish National } \\
\text { Health Survey }\end{array}$ & 1905 & Prevalence (CVD) \\
\hline Pérez [55] & $2011-2012$ & National & Cross-sectional & $\begin{array}{l}\text { Primary health } \\
\text { centres survey }\end{array}$ & 5382 & $\begin{array}{l}\text { Prevalence (CVD, } \\
\text { CHD, CVA, } \\
\text { PVD) }\end{array}$ \\
\hline Piniés [56] & $1998-2000$ & $\begin{array}{l}\text { Regional: } \\
\text { Basque } \\
\text { Country }\end{array}$ & $\begin{array}{l}\text { Population- } \\
\text { based, cross- } \\
\text { sectional }\end{array}$ & $\begin{array}{l}\text { Sentinel Practice } \\
\text { Network of } \\
\text { epidemiological } \\
\text { surveillance } \\
\text { database }\end{array}$ & 777 & $\begin{array}{l}\text { Prevalence (CVD, } \\
\text { CHD, CVA, } \\
\text { PAD); incidence } \\
\text { (CVD, CHD, } \\
\text { CVA, PAD); } \\
\text { mortality }\end{array}$ \\
\hline $\begin{array}{l}\text { Ramírez-Prado } \\
\text { [57] }\end{array}$ & $2010-2014$ & $\begin{array}{l}\text { Local: Hospital } \\
\text { del Elda } \\
\text { (Alicante) }\end{array}$ & $\begin{array}{l}\text { Retrospective } \\
\text { cohort }\end{array}$ & $\begin{array}{l}\text { Randomly selected } \\
\text { cohort }\end{array}$ & 112 & Mortality \\
\hline
\end{tabular}


Table 1 continued

\begin{tabular}{|c|c|c|c|c|c|c|}
\hline $\begin{array}{l}\text { First author } \\
\text { of article } \\
\text { [citation } \\
\text { number] }\end{array}$ & $\begin{array}{l}\text { Data } \\
\text { collection } \\
\text { period }\end{array}$ & $\begin{array}{l}\text { Geographic } \\
\text { range }\end{array}$ & Study design & Data source & $\begin{array}{l}\text { Patients } \\
\text { with } \\
\text { T2DM } \\
(N)\end{array}$ & $\begin{array}{l}\text { CV-related } \\
\text { outcomes }\end{array}$ \\
\hline Ramos [58] & $2005-2015$ & $\begin{array}{l}\text { Regional: } \\
\text { Catalonia }\end{array}$ & $\begin{array}{l}\text { Retrospective } \\
\text { cohort }\end{array}$ & $\begin{array}{l}\text { Regional database of } \\
\text { the primary care } \\
\text { system }\end{array}$ & 7880 & $\begin{array}{c}\text { Incidence (CVD, } \\
\text { CHD, CVA) }\end{array}$ \\
\hline $\begin{array}{l}\text { Rodríguez- } \\
\text { Poncelas } \\
\text { [59] }\end{array}$ & 2011 & National & Cross-sectional & $\begin{array}{l}\text { Primary health } \\
\text { centres }\end{array}$ & 674 & $\begin{array}{l}\text { Prevalence (CHD, } \\
\text { CVA, PVD) }\end{array}$ \\
\hline $\begin{array}{l}\text { Salinero-Fort } \\
\text { [60] }\end{array}$ & $2007-2012$ & $\begin{array}{l}\text { Local: Madrid } \\
\text { metropolitan } \\
\text { area }\end{array}$ & $\begin{array}{c}\text { Prospective } \\
\text { cohort }\end{array}$ & $\begin{array}{l}\text { Random sampling } \\
\text { from primary } \\
\text { health centres }\end{array}$ & 3407 & $\begin{array}{l}\text { Prevalence (CVD, } \\
\text { CHD, CVA, } \\
\text { PAD, HF); } \\
\text { mortality }\end{array}$ \\
\hline $\begin{array}{l}\text { Salinero-Fort } \\
\text { [61] }\end{array}$ & 2015 & $\begin{array}{l}\text { Regional: } \\
\text { Madrid } \\
\text { Community }\end{array}$ & $\begin{array}{l}\text { Population- } \\
\text { based, cross- } \\
\text { sectional }\end{array}$ & $\begin{array}{l}\text { Population study of } \\
\text { patients } \\
\text { aged } \geq 90 \text { years }\end{array}$ & 11,645 & $\begin{array}{l}\text { Prevalence (CVD, } \\
\text { CHD, CVA, } \\
\text { PAD, HF) }\end{array}$ \\
\hline $\begin{array}{l}\text { Sancho-Mestre } \\
\quad[62]\end{array}$ & 2012 & $\begin{array}{l}\text { Regional: } \\
\text { Valencia } \\
\text { Community }\end{array}$ & Cross-sectional & $\begin{array}{l}\text { Electronic health } \\
\text { records }\end{array}$ & 350,015 & $\begin{array}{l}\text { Prevalence (CVD); } \\
\text { costs } \\
\text { (pharmaceutical) }\end{array}$ \\
\hline $\begin{array}{l}\text { Sicras-Mainar } \\
\quad[63]\end{array}$ & $2008-2009$ & $\begin{array}{l}\text { Regional: } \\
\text { Badalona } \\
\text { (Catalonia) }\end{array}$ & $\begin{array}{l}\text { Retrospective } \\
\text { cohort }\end{array}$ & $\begin{array}{l}\text { Primary health care } \\
\text { survey }\end{array}$ & 987 & $\begin{array}{l}\text { Prevalence (CVD, } \\
\text { CHD, CVA) }\end{array}$ \\
\hline $\begin{array}{l}\text { Sicras-Mainar } \\
\text { [64] }\end{array}$ & 2010 & $\begin{array}{l}\text { Local: Badalona } \\
\text { (Catalonia) }\end{array}$ & $\begin{array}{l}\text { Retrospective } \\
\text { cohort }\end{array}$ & $\begin{array}{l}\text { Primary health care } \\
\text { survey }\end{array}$ & 3758 & $\begin{array}{l}\text { Incidence (CVD); } \\
\text { Costs }\end{array}$ \\
\hline Vinagre [65] & 2009 & $\begin{array}{l}\text { Regional: } \\
\text { Catalonia }\end{array}$ & $\begin{array}{l}\text { Retrospective } \\
\text { cohort }\end{array}$ & $\begin{array}{l}\text { Electronic health } \\
\text { records }\end{array}$ & 286,791 & $\begin{array}{l}\text { Prevalence (CHD, } \\
\text { CVA, PAD) }\end{array}$ \\
\hline $\begin{array}{l}\text { Zapatero- } \\
\text { Gaviria [66] }\end{array}$ & 2015 & National & Cross-sectional & $\begin{array}{l}\text { Hospital discharges } \\
\text { database: } \\
\text { minimum basic } \\
\text { data set of the } \\
\text { Spanish Ministry } \\
\text { of Health }\end{array}$ & 619,188 & $\begin{array}{l}\text { Prevalence (CVD, } \\
\text { CHD, CVA, } \\
\text { PAD, HF); } \\
\text { mortality }\end{array}$ \\
\hline
\end{tabular}

$\overline{A A A \text { abdominal aortic aneurysm, } A A D \text { aortic aneurysm and dissection, } A C S \text { acute coronary syndrome, } A L L I \text { acute lower }}$ limb ischemia, $A M I$ acute myocardial infarction, $C H D$ coronary heart disease, $C K D$ chronic kidney disease, $C V$ cardiovascular, $C V A$ cerebrovascular accident (stroke), $C V D \mathrm{CV}$ disease, $H b A 1 c$ glycated haemoglobin, $H F$ heart failure, $H R U$ health resource utilization, $P A D$ peripheral arterial disease, $T 2 D M$ type 2 diabetes mellitus 


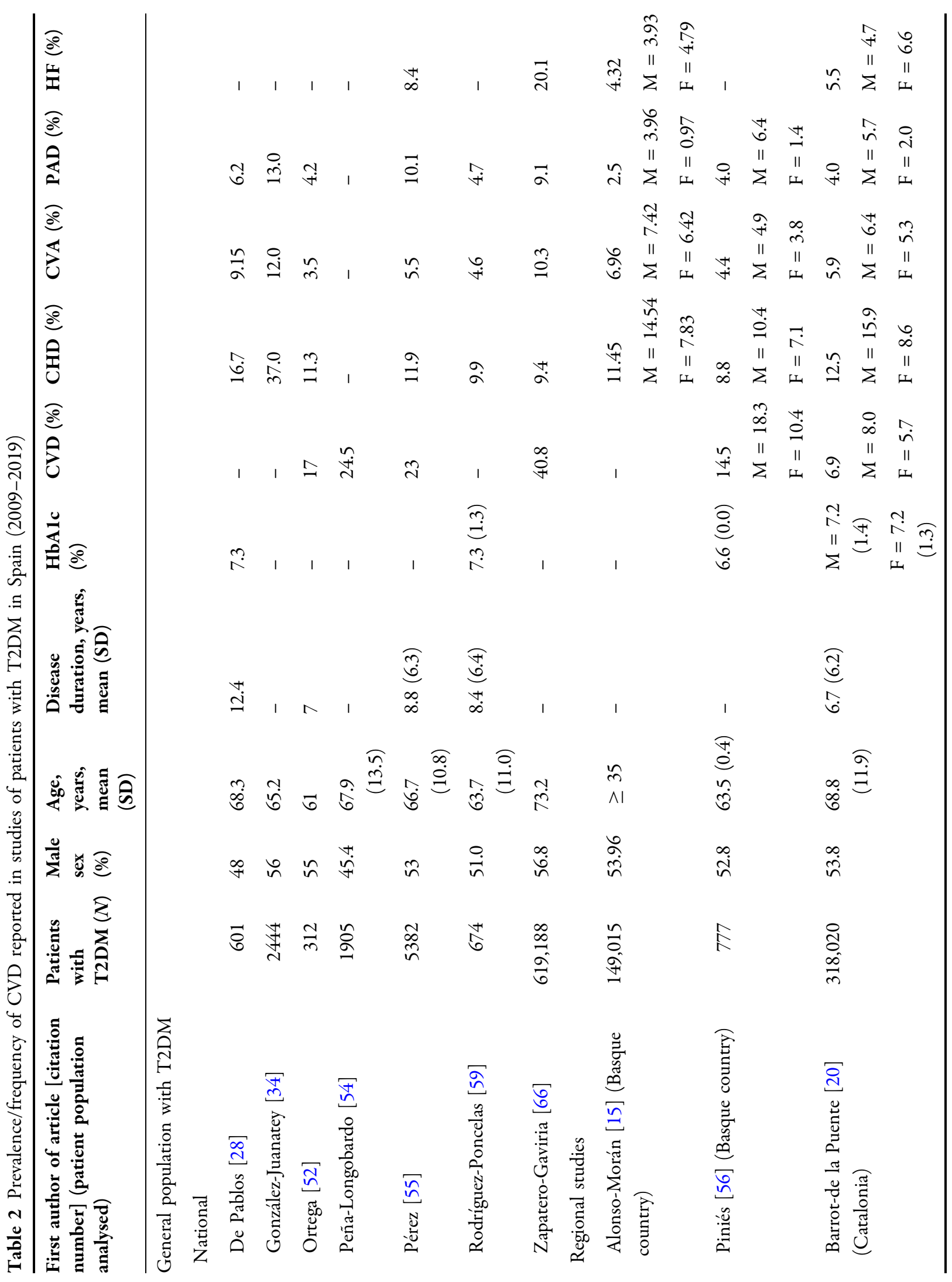




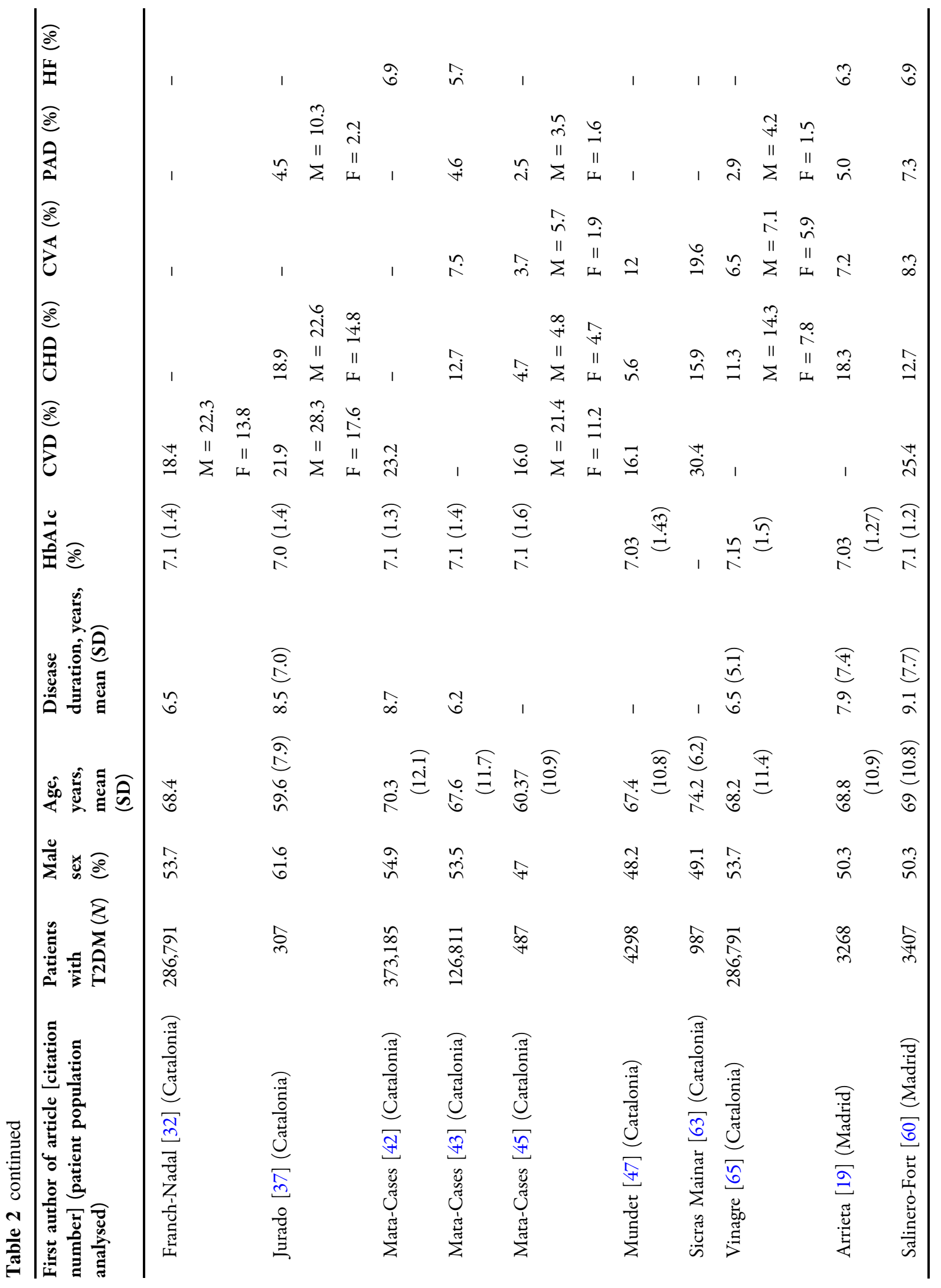




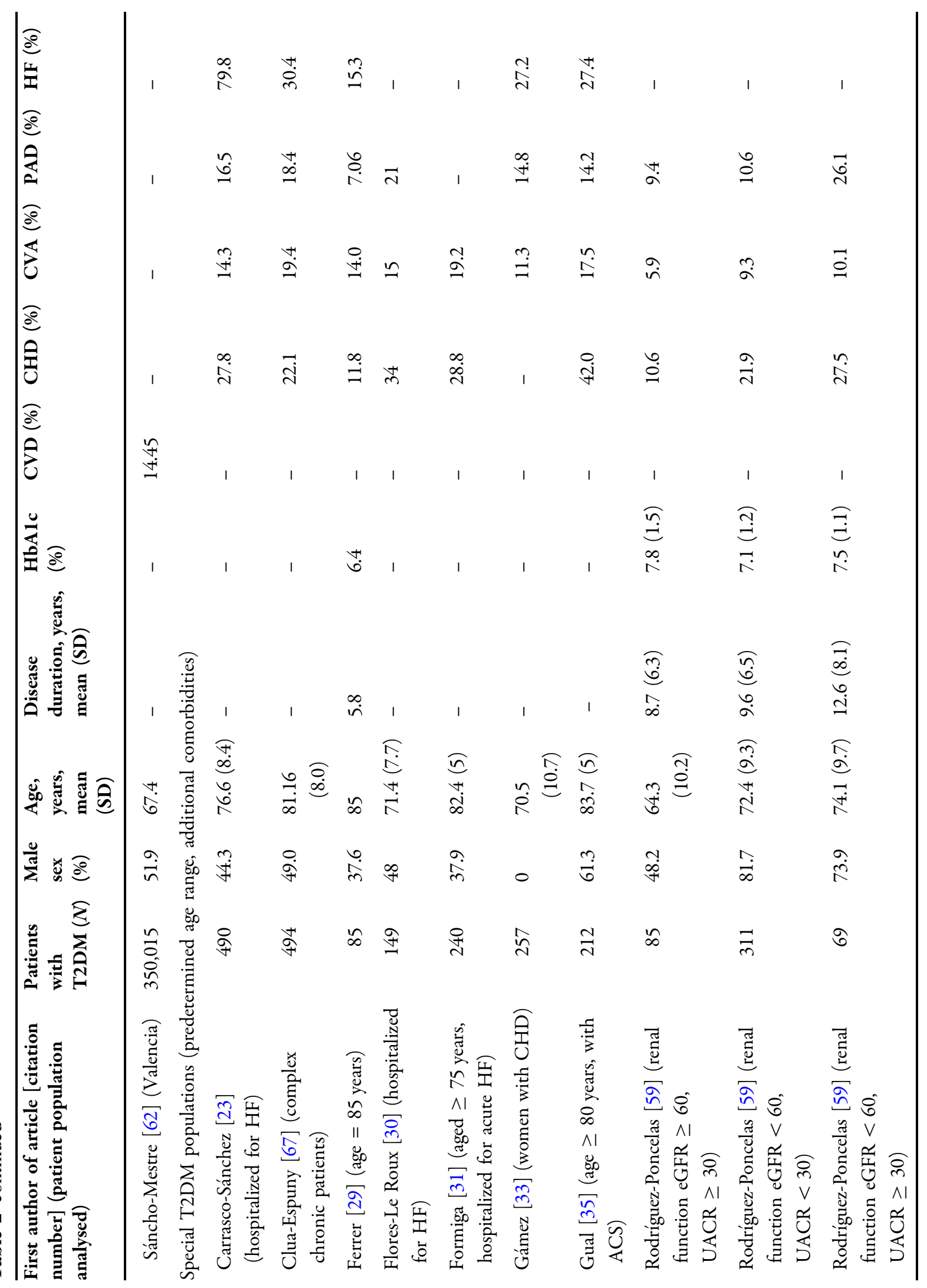




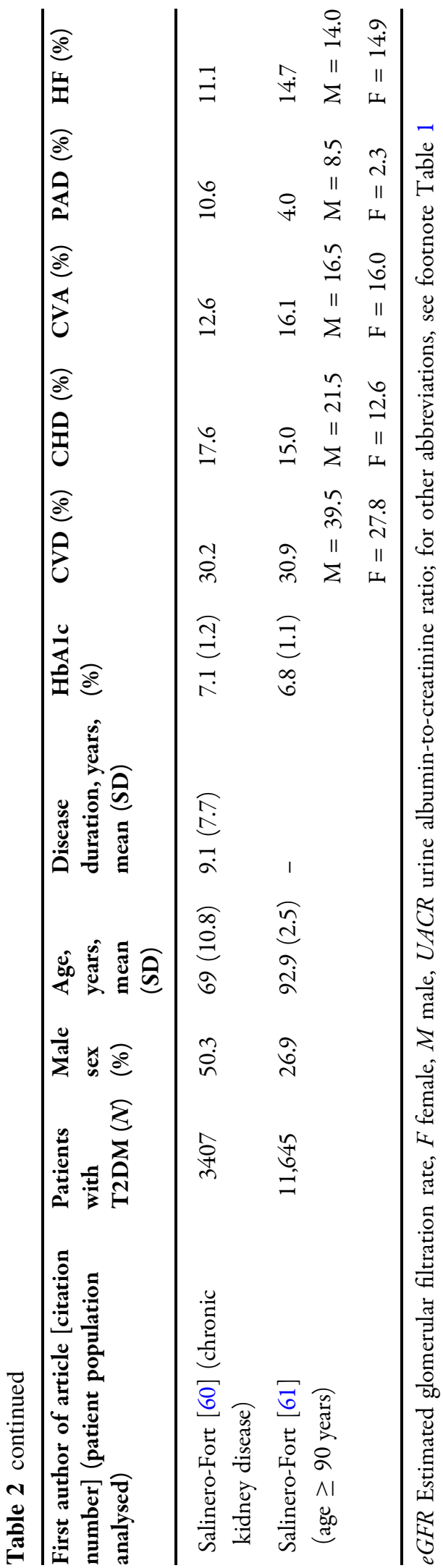

$[15,19,20,28,34,37,43,45,47,52$, $55,56,59,60,63,65,66] ; 3.5-19.6 \%$ for CVA (stroke; 16 studies) $[15,19,20,28,34,43,45$, $47,52,55,56,59,60,63,65,66] ; 2.5-13.0 \%$ for PAD (15 studies) $[15,19,20,28, \quad 34$, $37,43,45,52,55,56,59,60,65,66]$; and $4.3-20.1 \%$ for HF (eight studies) $[15,19,20$, $42,43,55,60,66]$.

Consistently, in the five studies that provided data on sex, men had a higher prevalence than women of CHD, CVA and PAD $[15,20,37,56,65]$.

The literature review identified several studies that reported the prevalence of CVD or a type of CVD in patients with T2DM in special populations, such as those of a predetermined age range or with additional comorbidities (Table 2). These studies focused on elderly patients $[29,31,35,61]$, patients with HF $[30,31,33,35]$ and patients with kidney disease $[59,60]$. Several studies reported age as one of the main risks for CVD in patients with T2DM. The prevalence of ischaemic heart disease, stroke and HF increased sharply with age in patients with T2DM [15]. Also, a large regional study in Catalonia showed that the prevalence of CVD was $32.3 \%$ in patients aged $\geq 75$ years and about 10 points lower in the overall population [42]. Two studies analysed the prevalence of CVD specifically in patients aged 85 and $\geq 90$ years, respectively $[29,61]$ : in one study, the prevalence of stroke was slightly higher among patients with T2DM aged 85 years $(14.0 \%)$ than in studies of the general population [29]; in the other study, the prevalence of CHD and PAD among nonagenarians with diabetes in the community of Madrid was higher in males than in females ( 21.5 vs. $12.6 \%$ and 8.5 vs. $2.3 \%$, respectively; both $p<0.01$ ) [61]. However, the prevalence of stroke in the latter study was similar in both sexes (16.5 vs. $16.0 \% ; p=0.44)$ [61].

Two studies analysed the prevalence of CVD in patients with T2DM and kidney disease $[59,60]$. The prevalence of CVD was high among patients with chronic kidney disease (30.2\%) [60] and increased dramatically with worsening estimated glomerular filtration rate (eGFR) and urine albumin-to-creatinine ratio (UACR) parameters. Decreases in eGFR and 


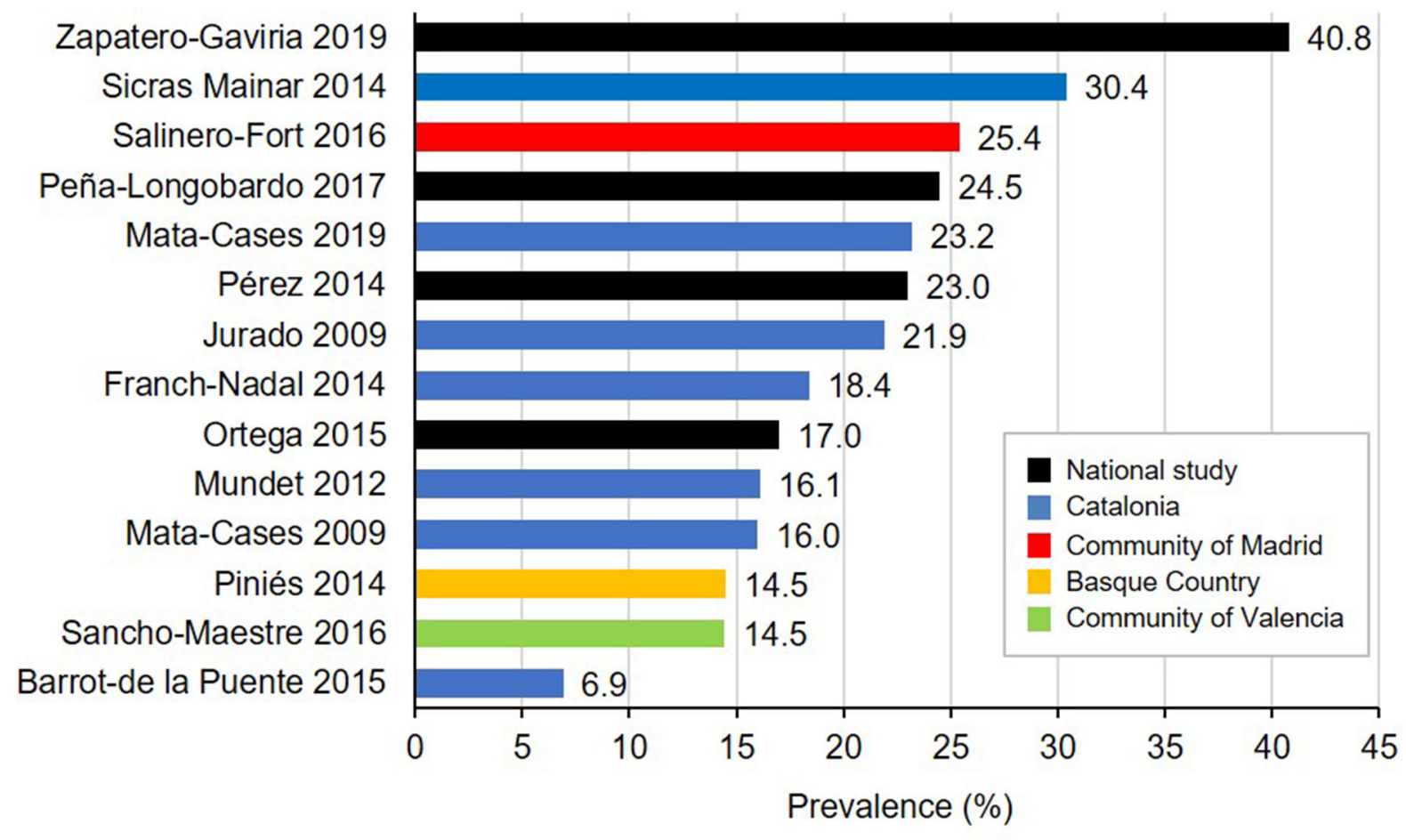

Fig. 2 Prevalence of CVD in patients with diabetes mellitus in national and regional studies published from 2009 to 2019. CVD Cardiovascular disease

increases in UACR are independent risk factors that increase the prevalence of CVD. The authors of the other study [59] suggested that both parameters should be determined at the time of T2DM diagnosis to identify patients at increased risk of CVD. The conclusion drawn in both studies was that patients with chronic kidney disease had an increased risk of $\mathrm{CV}$ mortality compared with patients without kidney disease $[59,60]$. A population-based study of comorbidities showed that the co-prevalence of chronic kidney disease and CVD among patients with T2DM was 19.3\% [42].

\section{Incidence of CV Events in Patients with T2DM}

The incidence of CVD or a type of CVD in patients with T2DM was analysed in 20 studies (Table 1). Several studies used the Spanish National Hospital Discharge Database (Registro de Altas de los Hospitales del Sistema Nacional de Salud [CMBD]) and other national databases to analyse the incidence of a specific CVD associated with patients with T2DM. These studies reported data on the incidence of acute myocardial infarction [27, 39, 40], thoracic aortic aneurysm [27, 36], ischaemic stroke $[27,50]$, haemorrhagic stroke [49], HF [48] and acute lower limb ischaemia [27] among patients with T2DM. Table 3 shows the incidence of CVD reported in studies among patients with T2DM in Spain. Most studies reported the incidence as rate per 100,000 inhabitants, but other formats were also used, which meant comparisons between studies were difficult.

The study with the most consistent and comprehensive data across all macrovascular comorbidities was that of de Miguel-Yanes et al. [27], which used data from the Spanish National Hospital Discharge Database. It also provided a time series for age-adjusted incidence from 2002 to 2014. For 2014, the most recent datapoint, this study reported an age-adjusted incidence of 145.13 per 100,000 inhabitants for all CVD combined [27]. 


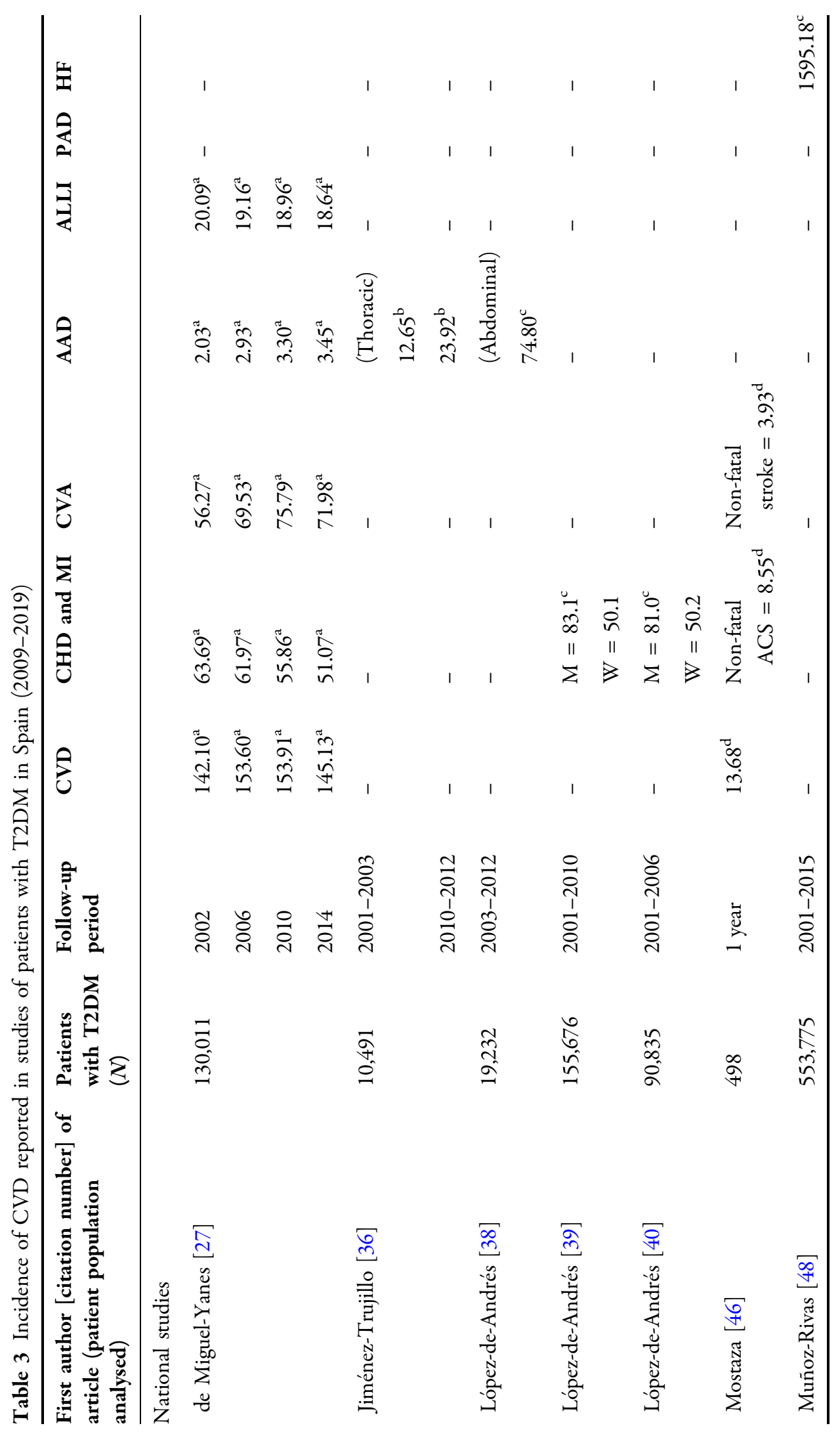




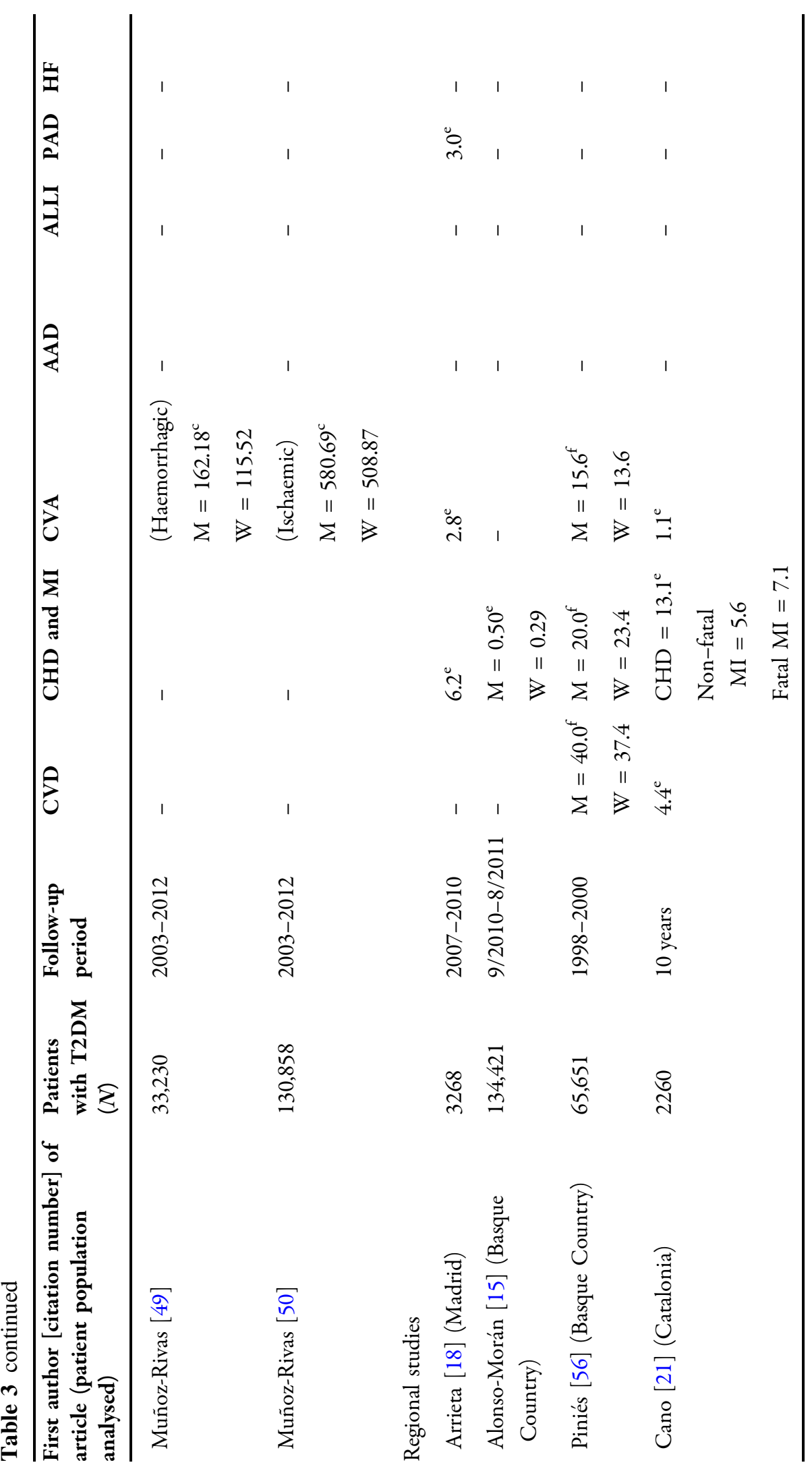




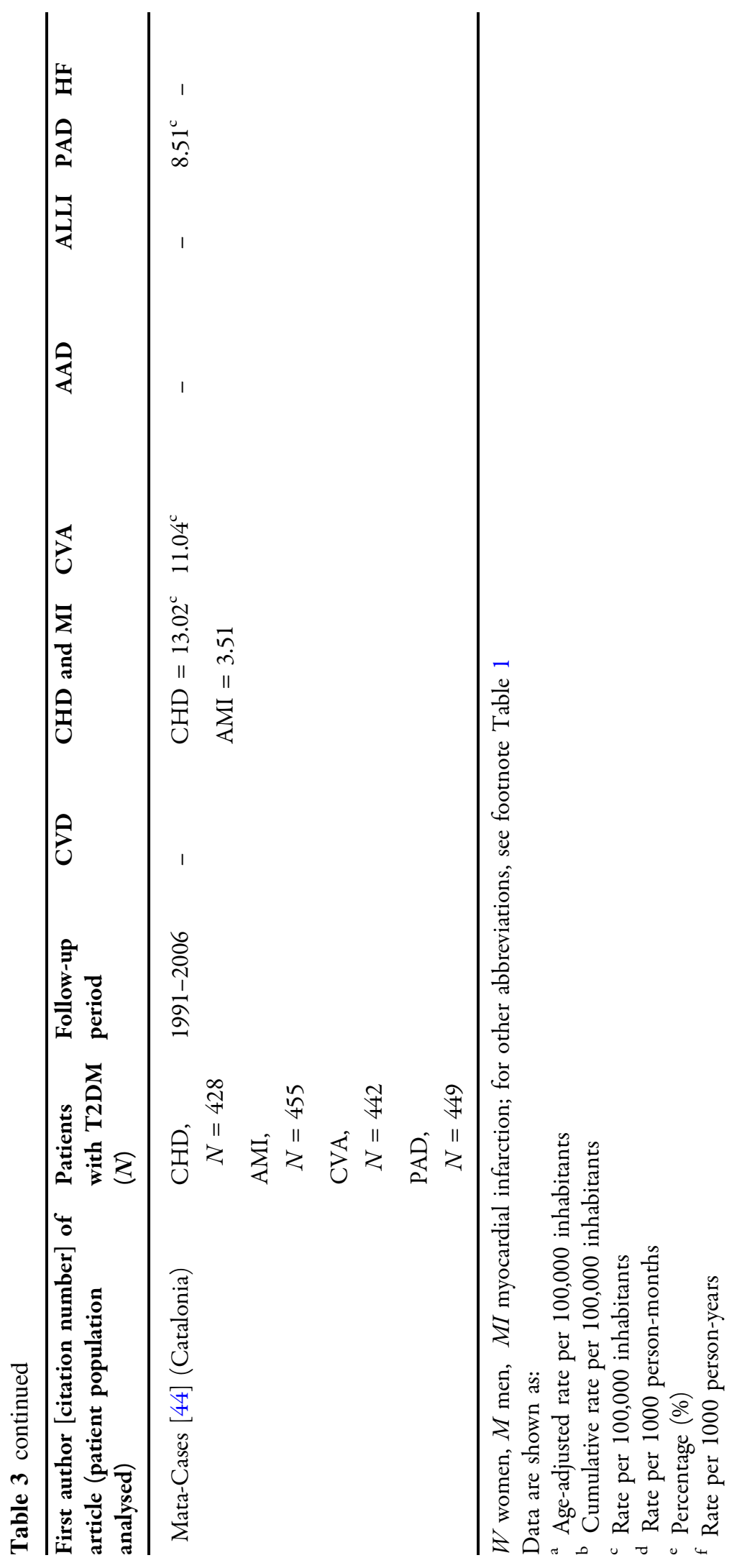


De Miguel-Yanes et al. [27] reported a trend of increasing incidence of stroke from 2002 to 2014 (56.27-71.98 per 100,000 inhabitants), whereas the incidence of CHD remained stable or declined slightly (63.69-51.07 per 100,000 inhabitants in that period) [27]. The regional studies by Mata Cases et al. [44] in Catalonia and Piniés et al. [56] in the Basque Country, for the periods 1991-2006 and 1998-2000, respectively, showed that the incidence of CHD was higher than that of stroke about two decades ago.

\section{Recurrence of CV Events in Patients with T2DM with Established CVD}

Information on the occurrence of subsequent CV events in patients with T2DM was specifically mentioned in 7 of the 20 studies reporting incidence $[22,23,30,31,33,35,60]$.

Camafort et al. [22] analysed the incidence of recurrent ischaemic events in a countrywide sample of 974 patients with T2DM prospectively enrolled in a registry of symptomatic artery disease. Over a mean follow-up of 14 months, $13 \%$ of the patients had a second ischaemic event. In patients with HbA1c levels $>7 \%$, the incidence rate was 4.4 (range 2.9-6.4) per 100 patient-years for myocardial infarction, $2.6(1.5-4.2)$ for acute ischemic stroke, and 6.7 (4.8-9.1) for critical limb ischaemia. Overall, the incidence rate of subsequent ischaemic events was 14 (11-17) per 100 patient-years [22]. This study concluded that for patients with coronary artery disease and HbA1c levels $<7.0 \%$, the incidence of subsequent ischaemic events was significantly reduced compared with patients with HbA1c levels $>7.0 \%$.

Salinero-Fort et al. [60] detailed the number of $\mathrm{CV}$ events in patients with T2DM from Madrid primary health care centres: $74.6 \%$ were free of CVD, $17.8 \%$ had one CV event, $6.8 \%$ had two CV events, and $1.3 \%$ had more than two $\mathrm{CV}$ events. Three studies reported previous CVD associated with patients with T2DM hospitalized for HF [23, 30, 31]. In patients with T2DM hospitalized for $\mathrm{HF}, 34 \%$ had previous $\mathrm{CHD}$, $15 \%$ had stroke, and $21 \%$ had PAD [30]. A second study in the same population showed that $79.8 \%$ had a previous episode of HF and $27.8 \%$ an episode of myocardial infarction [23].

Two studies studied subsequent CV events in elderly patients with T2DM [31,35]. A study of 240 patients aged $\geq 75$ years first admitted for acute HF reported that $28.8 \%$ had a previous diagnosis of CHD and $19.2 \%$ had a previous stroke [31]. Gual et al. [35] studied cases of acute coronary syndrome in patients aged $\geq 80$ years and found that a large proportion of patients had previous comorbid CVDs.

\section{Mortality for CVD in Patients with T2DM}

Mortality associated with CVD in patients with T2DM in Spain was analysed in 18 studies (Table 1). All used hospital registry data, and six of the seven studies at the national level reported data for CV-related mortality in patients with T2DM from the Spanish National Hospital Discharge Database [27, 38-40, 46, 48, 66]. Therefore, all of these studies presented data from hospitalized patients with T2DM.

Six studies analysed CV-related mortality in patients with T2DM in regional or local settings $[16,30,31,44,57,60]$. For these studies, the data sources were more diverse, with three population-based studies $[16,57,60]$, two studies based on hospital admissions [30, 31] and one study based on data from primary healthcare centres [44].

Table 4 shows the mortality rates obtained in 13 of the 18 studies analysing mortality (data from the remaining five studies were not tabulated as they only reported hazard ratios or mortality in a subpopulation). Mortality ranged from $5.6 \%$ [46] to $10.8 \%$ [27]. A recent study found that CVD-related in-hospital mortality in patients with T2DM declined significantly in the years analysed (2002-2014) [27]. However, multivariate analysis showed that, compared with matched controls without diabetes, patients with T2DM had a 15, 6 and $6 \%$ higher mortality when admitted for myocardial infarction, CVA and CVD, respectively [27]. 
Table 4 Mortality due to CVD of patients with T2DM in Spain (2009-2019)

\begin{tabular}{|c|c|c|c|c|c|c|c|c|c|}
\hline $\begin{array}{l}\text { First author [citation } \\
\text { number] of article } \\
\text { (patient population } \\
\text { analysed) }\end{array}$ & $\begin{array}{l}\text { Patients } \\
\text { with } \\
\text { T2DM } \\
(N)\end{array}$ & $\begin{array}{l}\text { Follow-up } \\
\text { period }\end{array}$ & CVD & $\begin{array}{l}\text { CHD and } \\
\text { MI }\end{array}$ & CVA & AAD & ALLI & PAD & $\mathbf{H F}$ \\
\hline \multicolumn{10}{|l|}{ National studies } \\
\hline \multirow[t]{4}{*}{ De Miguel-Yanes [27] } & 130,011 & 2002 & $13.04^{\mathrm{a}}$ & $13.67^{\mathrm{a}}$ & $14.97^{\mathrm{a}}$ & $14.36^{\mathrm{a}}$ & $5.53^{\mathrm{a}}$ & - & - \\
\hline & & 2006 & $12.41^{\mathrm{a}}$ & 10.83 & $15.49^{\mathrm{a}}$ & $12.52^{\mathrm{a}}$ & $6.34^{\mathrm{a}}$ & - & - \\
\hline & & 2010 & $11.78^{\mathrm{a}}$ & $9.49^{a}$ & $14.94^{\mathrm{a}}$ & $10.89^{\mathrm{a}}$ & $6.04^{\mathrm{a}}$ & - & - \\
\hline & & 2014 & $10.77^{\mathrm{a}}$ & $7.95^{\mathrm{a}}$ & $14.27^{\mathrm{a}}$ & $8.09^{\mathrm{a}}$ & $5.47^{\mathrm{a}}$ & - & - \\
\hline López-de-Andrés [38] & 19,232 & $2012-2013$ & - & - & - & $\begin{array}{l}\text { (Abdominal) } \\
9.37^{\mathrm{a}}\end{array}$ & - & - & - \\
\hline López-de-Andrés [39] & 155,676 & $2001-2010$ & - & $\begin{array}{l}M=9.4^{\mathrm{a}} \\
W=14.9\end{array}$ & - & - & - & - & - \\
\hline López-de-Andrés [40] & 90,835 & $2001-2006$ & - & $\begin{array}{l}\mathrm{M}=10.1^{\mathrm{a}} \\
\mathrm{W}=15.9\end{array}$ & - & - & - & - & - \\
\hline Mostaza [46] & 498 & 1 year & $5.6^{\mathrm{a}}$ & - & - & - & - & - & - \\
\hline Muñoz-Rivas [48] & 553,775 & $2001-2015$ & - & - & - & - & - & - & $8.35^{\mathrm{a}}$ \\
\hline Zapatero-Gaviria [66] & 619,188 & 2015 & $6.8^{\mathrm{a}}$ & - & - & - & - & - & - \\
\hline \multicolumn{10}{|l|}{ Regional studies } \\
\hline Cano [21] (Catalonia) & 2260 & 10 year & $4.4^{\mathrm{a}}$ & $1.8^{\mathrm{a}}$ & $1.1^{\mathrm{a}}$ & - & - & - & - \\
\hline $\begin{array}{l}\text { Formiga }[31] \\
\text { (Catalonia, patients } \\
\text { aged } \geq 75 \text { years })\end{array}$ & 240 & 1 year & - & - & - & - & - & - & $5.0^{\mathrm{a}}$ \\
\hline $\begin{array}{l}\text { Mata-Cases [44] } \\
\text { (Catalonia) }\end{array}$ & 469 & - & $19.23^{b}$ & - & - & - & - & - & - \\
\hline $\begin{array}{l}\text { Piniés [56] (Basque } \\
\text { Country) }\end{array}$ & 65,651 & $1998-2000$ & $\begin{array}{l}\mathrm{M}=10.6^{\mathrm{b}} \\
\mathrm{W}=13.0\end{array}$ & - & - & - & - & - & - \\
\hline $\begin{array}{l}\text { Ramírez-Prado [57] } \\
\text { (Valencia) }\end{array}$ & 112 & $2010-2014$ & $19.6^{\mathrm{a}}$ & - & - & - & - & - & - \\
\hline $\begin{array}{l}\text { Salinero-Fort [60] } \\
\text { (Madrid) }\end{array}$ & 3443 & $2008-2012$ & $13.7^{b}$ & & & & & & \\
\hline
\end{tabular}

All data refer to in-hospital mortality, with the exception of data from Cano et al. [21] (Catalonia) and Salinero-Fort et al. [60] (Madrid)

$W$ women, $M$ men, $M I$ myocardial infarction; for other abbreviations, see footnote Table 1

Data are shown as:

a Percentage (\%)

b Rate per 1000 patients/year 
Table 5 Studies analysing HRU and costs associated with CVD in adults with T2DM in Spain (2009-2019)

\begin{tabular}{lllllllll}
\hline $\begin{array}{l}\text { First author of article [citation } \\
\text { number] (national or regional) }\end{array}$ & $\begin{array}{l}\text { Year for cost } \\
\text { calculation }\end{array}$ & $\begin{array}{l}\text { Patients } \\
\text { with T2DM } \\
(\boldsymbol{N})\end{array}$ & CVD & CHD & AMI & CVA & PAD & HF \\
\hline Crespo [26] (National) & 2012 & 544,515 & - & $5587^{\mathrm{a}, \mathrm{b}}-$ & $5319^{\mathrm{a}, \mathrm{b}}$ & $6875^{\mathrm{a}, \mathrm{b}}$ & - \\
López-de-Andrés [40] (National) & 2006 & 90,835 & - & - & $6228^{\mathrm{b}, \mathrm{c}}$ & - & - & - \\
Arrieta [17] (Madrid) & 2012 & 3268 & $2726.50^{\mathrm{a}}-$ & - & - & - & - \\
Sancho-Mestre [62] (Valencia) & 2012 & 350,015 & $1441^{\mathrm{a}, \mathrm{d}}$ & - & - & - & - & - \\
Mata-Cases [43] (Catalonia) & 2011 & 126,811 & $4815^{\mathrm{c}}$ & $5006^{\mathrm{c}}-$ & $4720^{\mathrm{c}}$ & $5858^{\mathrm{c}}$ & $6866^{\mathrm{c}}$ \\
\hline
\end{tabular}

For abbreviations, see footnote Table 1

All costs are expressed in euros

a Mean cost per episode per patient

b Hospitalization costs

c Total cost per patient and year

d Pharmaceutical costs

\section{Costs Associated with CVD in Patients with T2DM}

Five studies analysed the costs of CV complications in patients with T2DM (Table 1). All studies identified in this review reported only direct costs from CV comorbidity in patients with T2DM.

Two studies analysed economic costs at the national level $[26,40]$, and three analysed costs at the regional level in Madrid [17], Catalonia [43], and Valencia [62]. Costs reported in these studies referred to the years 2006-2012.

Table 5 shows data from the five studies included in this review. Mata-Cases et al. [43] estimated the annual cost per patient with T2DM as $€ 3110$ (year 2011 values), but the presence of CVD increased costs to $€ 4814$. The largest increase in costs was generated by $\mathrm{HF}$, which reached $€ 6866$ per year in patients with T2DM, followed by PAD ( $€ 5858$ ), CHD (€5006), and stroke $(€ 4720)$ [43]. A study of the costs of complications of T2DM in Madrid found a mean overall cost of $€ 4121$, of which two-thirds was due to macrovascular complications (especially heart revascularization and acute myocardial infarction) [17].

None of these studies analysed CVD cost components comprehensively, as some studies only evaluated hospitalization costs $[26,40]$ and others only medication costs [62]. Generally, costs were driven mainly by hospitalizations and medications, and CVD caused an increase in both components [43].

\section{DISCUSSION}

In this systematic review we evaluated the epidemiology and costs of CVD associated with T2DM in Spain reported in studies published between 2009 and 2019. To the best of our knowledge, this is the first systematic review of studies of epidemiology and costs in this patient group in Spain. The overall prevalence of CVD in Spain in this period ranged from 7 to $41 \%$. This range is consistent with the global prevalence of $32.2 \%$ found in a systematic literature review of articles from across the world in approximately the same period (2007-2017) [1]. However, despite the relatively large number of studies included, the diversity of methodologies, populations analysed and data reporting made it difficult to reach quantitative conclusions, as discussed in the following text.

Current guidelines highlight the importance of early prevention to reduce the risks of CVD in patients with T2DM $[5,67]$. It has been estimated that patients with T2DM have a two- to fourfold higher risk of developing CVD and that this increased risk correlates directly with the 
number and control of CV risk factors, including the level of glycaemic control [68, 69]. For this reason, the expanded use of therapies to reduce CVD risk in the T2DM population prior to the development of CVD has been advocated [70]. Generally, CVD risk management in patients with T2DM has focused on the use of aspirin and the control of blood pressure, cholesterol and glycaemia. Although most benefit is obtained when multiple CVD risk factors are addressed simultaneously, the greatest absolute risk reduction, especially for microvascular complications, is derived from improving glycaemic control [5]. In this regard, some antidiabetic drugs with proven CV benefit, such as the SLGT2i and the GLP-1 RAs, are recommended for the prevention of $\mathrm{CV}$ events in patients with established CVD or at high CV risk [6]. The strongest evidence to date is with GLP-1 RAs, specifically dulaglutide, for which an added benefit in early primary prevention has been reported $[6,71]$.

Although preventing the recurrence of $\mathrm{CV}$ events in patients with T2DM is a major objective in those with established CVD, epidemiological data related to this topic are limited. We identified seven studies with relevant data on recurrence of CVD in patients with T2DM in Spain. Two independent studies agreed that patients with T2DM hospitalized for HF had a high prevalence $(>70 \%)$ of established CVD [23, 30]. Camafort et al. [22] found that patients with T2DM with CHD and glycated haemoglobin (HbA1c) levels $<7.0 \%$ had a lower incidence of subsequent ischaemic events and lower mortality rates than those with HbA1c levels $>7.0 \%$, highlighting the importance of glycaemic control [22]. However, these authors reported no difference in HbA1c levels for patients with CVA or PAD. Factors independently associated with a lower risk for recurrent events were lower age ( $<70$ years), mean HbA1c levels $<7 \%, \quad$ creatinine clearance levels $>60 \mathrm{ml} / \mathrm{min}$ and the use of statins [22]. Studies in other countries have also found that recurrent $\mathrm{CV}$ events were associated with higher HbA1c levels [72, 73], suggesting that some groups of patients with CVD could benefit from more intensive glycaemic control. Along these lines, the Steno-2 randomized trial showed that intensive, multifactorial management of hyperglycaemia, hypercholesterolaemia, and hypertension, together with antiplatelet therapy, could lead to significant reductions in CVD and mortality rates in the T2DM population, with a mean increase in life expectancy of 7.9 years after a follow-up period of 21 years [74].

CVD is a major risk factor for patients with T2DM and has been associated with increased mortality rates compared with patients with T2DM without CVD. In a review compiling data from across the world, CVD was found to be responsible for $50.3 \%$ of all deaths of patients with T2DM, with CHD and stroke being the major contributors [1]. A study in a UK population found myocardial infarction to be the leading cause of death among those with T2DM [75]. The studies included in our review suggest that the current in-hospital mortality rate in Spain could range between 6 and $11 \%$, which is consistent with the rate of $9.9 \%$ found in the Einarson et al. review [1]. However, CVD-related mortality in the T2DM population in the USA and Europe has been declining in the last few decades [76, 77]. This trend is consistent with the data series for Spain described by de MiguelYanes et al. [27], which showed a progressive decrease of in-hospital mortality by CVD in patients with T2DM from 2002 to 2014 [27].

Interestingly, Muñoz-Rivas et al. [48] found that in-hospital mortality in patients due to HF was lower in patients with T2DM than in those without T2DM, a result distinct from other results reported in the literature [78]. Also, in three studies reporting data on the association between sex and in-hospital mortality, women had higher in-hospital mortality than men $[38,39,56]$.

In this systematic literature review, a few articles included data on HRU and cost-related variables related to CVD in patients with T2DM in Spain. A study of patients with T2DM in the USA found that CVD dramatically increased costs by $70-150 \%$ [79], and a recent review of costs in multiple countries found that the median annual costs per patient for CVD, coronary artery disease, HF and stroke were, respectively, 112, 107, 59 and 322\% higher than 
in those for patients with T2DM without CVD [12].

For Spain, the most complete study, by MataCases et al. [43] in Catalonia, found that CVD increased direct costs for patients with T2DM by 58\% compared with patients with T2DM with no CVD, and other studies have also emphasized the very high increases $(>50 \%)$ in associated costs [17]. To date, no studies have evaluated the indirect costs of CVD in a T2DM population. A review of studies of T2DM-related costs estimated that the total direct costs of macrovascular complications in Spain in 2009 were $€ 757.74$ million, of which $44.1 \%$ were for inpatient care (hospitalization), $34.8 \%$ were for pharmaceuticals and $21.1 \%$ were for outpatient care [80]. A review by the Health Technologies Assessment Agency of Spain confirmed that the major components of the costs of T2DM were hospitalization (10-67\%) and costs of medication for T2DM complications (14-33\%) [81], results also reported by the previously mentioned Mata-Cases et al. study in Catalonia [43]. Further, a recent study demonstrated that the length of hospital stay was longer for patients with T2DM, compared with patients without diabetes, for non-fatal acute myocardial infarction and unstable angina, resulting in higher hospitalization costs [82].

Given the high costs of macrovascular complications in T2DM in Spain, and considering the high risk of CVD development, early prevention seems critical. The early use of pharmacological therapies in T2DM that have shown CVD benefit could directly and positively mitigate the high medical costs of treating diabetes complications later on [6].

\section{Limitations}

The wide heterogeneity of the included studies was a major obstacle to pooling data and defining more precise epidemiological outcomes. A main source of variation and possible bias was the epidemiological data sources used. A large number of studies reported data from hospital discharge databases, but others relied on surveys from primary healthcare centres and other sources. Although CVDs are serious and usually require patient hospitalization at some point in their lifetime, hospital discharge data could represent a population that is in worse medical condition and therefore not representative of the general T2DM population. In turn, this could lead to an overestimation of the prevalence of CVD in patients with T2DM. Additionally, some studies included only predefined populations that could be biased with respect to the general T2DM population, such as patients with newly diagnosed T2DM [24] or patients admitted to only a specific unit in the hospital [30].

A second major source of variation was the range of definitions used to assess CVD (ESM Table S1). Although most studies used the World Health Organization ICD to identify types of CVD, some articles did not specify the codes included, others included wide ranges of the classification (e.g., all ICD-9 codes from I00 to I99) and still others were highly restrictive in the specific pathologies included. As such, for some articles it was not possible to determine whether global CVD outcomes included or excluded microvascular diabetes complications (diabetes retinopathy, nephropathy or neuropathy). Also, it is possible that studies with data from primary healthcare centres included microvascular diabetes complications. Although the impact of microvascular comorbidities can be lower than that of macrovascular comorbidities, they are more prevalent, and their inclusion in studies could therefore affect the statistics.

The large hospital databases in Spain, notably the National Hospital Discharge Database (Registro de Atención Especializada-Conjunto Mínimo Básico de Datos [RAE-CMBD]), have been the basis for numerous epidemiological studies at the national level. Regional databases are also available for some autonomous communities and have served as the source of data for those territories. The Catalonia region had the largest number of studies $(N=18)$, but most of the Spanish autonomous communities had no studies conducted in their territories. This could have caused some degree of bias towards certain areas of the country, as significant differences in the epidemiology of T2DM and CVD 
have previously been found between autonomous communities [83, 84].

Another source of variation was the time period considered. Although we restricted study publication to the years 2009-2019 (inclusive), data were collected from the years 1991-2016. This could notably bias results, as some incidence studies with long follow-up periods observed increasing or decreasing trends for some conditions [27]. Finally, a lack of uniformity in reporting data, such as the units used to describe incidence, made it difficult to compare the results of some studies.

\section{CONCLUSIONS}

This systematic review evaluated the epidemiology and costs of CVD in adults with T2DM in Spain from studies published between January 2009 and December 2019. The results indicate that $7-41 \%$ of patients with T2DM also have CVD, with CHD the most prevalent CVD. In this regard, some studies suggest a decline of inhospital mortality over time, as observed in other Western countries, although CVD remains the leading cause of mortality among patients with T2DM. CVD causes major increases in costs compared with patients with T2DM without CVD. Given the major impact of chronic diseases such as T2DM on the healthcare system, further studies should be carried out to update the direct economic costs and evaluate the indirect costs associated with T2DM comorbidities. Our results highlight the importance of early prevention of CVD in patients with T2DM as part of integrated management of the disease to improve clinical and economic outcomes.

\section{ACKNOWLEDGEMENTS}

Funding. This research and the journal's Rapid Service Fee were funded by Lilly and Company.

Medical Writing Assistance. Medical Writing assistance was provided by Francisco López de Saro and Fernando Rico-Villademoros (Rx Communications, Mold, UK), funded by Lilly and Company.

Authorship. All named authors meet the International Committee of Medical Journal Editors (ICMJE) criteria for authorship for this article, take responsibility for the integrity of the work as a whole, and have given their approval for this version to be published.

Disclosures. Esther Artime, Irene Romera, and Silvia Díaz-Cerezo are employees of Lilly and Company. Esther Artime is a stock shareholder in Eli Lilly and Company. Elías Delgado declares no conflict of interest. The funder had no role in the design of the study; in the collection, analyses, or interpretation of data; in the writing of the manuscript, or in the decision to publish the results.

Compliance with Ethics Guidelines. This article is based on previously conducted studies and does not contain any new studies with human participants or animals performed by any of the authors.

Data Availability. All data generated analysed during this study are included in this published article/as supplementary information files.

Open Access. This article is licensed under a Creative Commons Attribution-NonCommercial 4.0 International License, which permits any non-commercial use, sharing, adaptation, distribution and reproduction in any medium or format, as long as you give appropriate credit to the original author(s) and the source, provide a link to the Creative Commons licence, and indicate if changes were made. The images or other third party material in this article are included in the article's Creative Commons licence, unless indicated otherwise in a credit line to the material. If material is not included in the article's Creative Commons licence and your intended use is not permitted by statutory regulation or exceeds the permitted use, you will need to obtain permission directly from the copyright holder. To view 
a copy of this licence, visit http:// creativecommons.org/licenses/by-nc/4.0/.

\section{REFERENCES}

1. Einarson TR, Acs A, Ludwig C, Panton UH. Prevalence of cardiovascular disease in type 2 diabetes: a systematic literature review of scientific evidence from across the world in 2007-2017. Cardiovasc Diabetol. 2018;17:83.

2. IDF. International Diabetes Federation: diabetes and cardiovascular disease. 2019. http://www.idf. org. Accessed 15 Jan 2020.

3. Low Wang CC, Hess CN, Hiatt WR, Goldfine AB. Clinical update: cardiovascular disease in diabetes mellitus: atherosclerotic cardiovascular disease and heart failure in type 2 diabetes mellitus-mechanisms, management, and clinical considerations. Circulation. 2016;133:2459-502.

4. Bashier A, Bin Hussain A, Abdelgadir E, Alawadi F, Sabbour H, Chilton R. Consensus recommendations for management of patients with type 2 diabetes mellitus and cardiovascular diseases. Diabetol Metab Syndr. 2019;11:80.

5. Davies MJ, D'Alessio DA, Fradkin J, et al. Management of hyperglycaemia in type 2 diabetes, 2018. A consensus report by the American Diabetes Association (ADA) and the European Association for the Study of Diabetes (EASD). Diabetologia. 2018;61: 2461-98.

6. Buse JB, Wexler DJ, Tsapas A, et al. 2019 Update to: management of hyperglycemia in type 2 diabetes, 2018. A consensus report by the American Diabetes Association (ADA) and the European Association for the Study of Diabetes (EASD). Diabetes Care. 2020;43:487-93.

7. Hudspeth B. The burden of cardiovascular disease in patients with diabetes. Am J Manag Care. 2018;24:S268-72.

8. Garber AJ, Handelsman Y, Grunberger G, et al. Consensus statement by the American Association of Clinical Endocrinologists and American College of Endocrinology on the comprehensive type 2 diabetes management algorithm-2020 executive summary. Endocr Pract. 2020;26:107-39.

9. Reyes-Garcia R, Moreno-Perez O, Tejera-Pérez C, et al. Sociedad Española de Endocrinología y Nutrición. Documento de abordaje integral de la DM2. Grupo de trabajo de diabetes de la SEEN (Versión 2019.2). 2019. https://www.seen.es/docs/
apartados/791/Abordaje\%20Integral\%20DM2_ SEEN_2019_OCT_ISBN\%20.pdf. Accessed 6 June 2020.

10. Vetrone LM, Zaccardi F, Webb DR, et al. Cardiovascular and mortality events in type 2 diabetes cardiovascular outcomes trials: a systematic review with trend analysis. Acta Diabetol. 2019;56:331-9.

11. Kant R, Munir KM, Kaur A, Verma V. Prevention of macrovascular complications in patients with type 2 diabetes mellitus: review of cardiovascular safety and efficacy of newer diabetes medications. World J Diabetes. 2019;10:324-32.

12. Einarson TR, Acs A, Ludwig C, Panton UH. Economic burden of cardiovascular disease in type 2 diabetes: a systematic review. Value Health. 2018;21:881-90.

13. Larsson SC, Wallin A, Hakansson N, Stackelberg O, Back M, Wolk A. Type 1 and type 2 diabetes mellitus and incidence of seven cardiovascular diseases. Int J Cardiol. 2018;262:66-70.

14. Shah AD, Langenberg C, Rapsomaniki E, et al. Type 2 diabetes and incidence of cardiovascular diseases: a cohort study in 1.9 million people. Lancet Diabetes Endocrinol. 2015;3:105-13.

15. Alonso-Morán E, Orueta JF, Fraile Esteban JI, et al. The prevalence of diabetes-related complications and multimorbidity in the population with type 2 diabetes mellitus in the Basque Country. BMC Public Health. 2014;14:1059.

16. Ares J, Valdes $\mathrm{S}$, Botas $\mathrm{P}$, et al. Mortality risk in adults according to categories of impaired glucose metabolism after 18 years of follow-up in the North of Spain: the Asturias study. PLoS ONE. 2019;14: e0211070.

17. Arrieta F, Rubio-Terres C, Rubio-Rodriguez D, et al. Estimation of the economic and health impact of complications of type 2 diabetes mellitus in the autonomous community of Madrid (Spain). Endocrinol Nutr. 2014;61:193-201.

18. Arrieta F, Pinera M, Iglesias $\mathrm{P}$, et al. Metabolic control and chronic complications during a 3-year follow-up period in a cohort of type 2 diabetic patients attended in primary care in the Community of Madrid (Spain). Endocrinol Nutr. 2014;61: 11-7.

19. Arrieta F, Salinero $M$, Piñera $M$, et al. Estudio descriptivo de la evolución clínico-asistencial de la población con diabetes tipo 2 en la Comunidad de Madrid. Estudio de seguimiento diabético tipo 2 (ESD-2). Av En Diabetol. 2011;27:53-60. 
20. Barrot-de la Puente J, Mata-Cases M, Franch-Nadal $\mathrm{J}$, et al. Older type 2 diabetic patients are more likely to achieve glycaemic and cardiovascular risk factors targets than younger patients: analysis of a primary care database. Int J Clin Pract. 2015;69:1486-95.

21. Cano JF, Baena-Diez JM, Franch J, et al. Long-term cardiovascular risk in type 2 diabetic compared with nondiabetic first acute myocardial infarction patients: a population-based cohort study in southern Europe. Diabetes Care. 2010;33:2004-9.

22. Camafort M, Alvarez-Rodriguez LR, Munoz-Torrero $\mathrm{JF}$, et al. Glucose control and outcome in patients with stable diabetes and previous coronary, cerebrovascular or peripheral artery disease. Findings from the FRENA Registry. Diabet Med. 2011;28: 73-80.

23. Carrasco-Sanchez FJ, Gomez-Huelgas R, Formiga F, et al. Association between type-2 diabetes mellitus and post-discharge outcomes in heart failure patients: findings from the RICA registry. Diabetes Res Clin Pract. 2014;104:410-9.

24. Catalan M, Herreras Z, Pinyol M, et al. Prevalence by sex of preclinical carotid atherosclerosis in newly diagnosed type 2 diabetes. Nutr Metab Cardiovasc Dis. $2015 ; 25: 742-8$.

25. Clua-Espuny JL, Gonzalez-Henares MA, QueraltTomas MLL, et al. Mortality and cardiovascular complications in older complex chronic patients with type 2 diabetes. Biomed Res Int. 2017;2017: 6078498.

26. Crespo C, Brosa M, Soria-Juan A, López-Alba A, López-Martínez N, Soria B. Direct cost of diabetes mellitus and its complications in Spain (SECCAID Study: Spain estimated cost Ciberdem-Cabimer in Diabetes). Av Diabetol. 2013;29:182-9.

27. de Miguel-Yanes JM, Jimenez-Garcia R, HernandezBarrera V, Mendez-Bailon M, de Miguel-Diez J, Lopez-de-Andres A. Impact of type 2 diabetes mellitus on in-hospital-mortality after major cardiovascular events in Spain (2002-2014). Cardiovasc Diabetol. 2017;16:126.

28. de Pablos Velasco P, Franch J, Banegas Banegas JR, Fernandez Anaya S, Sicras Mainar A, Diaz Cerezo S. Cross-sectional epidemiological study of clinical profiles and glycemic control in diabetic patients in primary care in Spain (the EPIDIAP study). Endocrinol Nutr. 2009;56:233-40.

29. Ferrer A, Padros G, Formiga F, Rojas-Farreras S, Perez JM, Pujol R. Diabetes mellitus: prevalence and effect of morbidities in the oldest old. The Octabaix study. J Am Geriatr Soc. 2012;60:462-7.
30. Flores-Le Roux JA, Comin J, Pedro-Botet J, et al. Seven-year mortality in heart failure patients with undiagnosed diabetes: an observational study. Cardiovasc Diabetol. 2011;10:39.

31. Formiga F, Chivite D, Montero A, et al. Association between diabetes and mortality in elderly patients admitted for a first episode of acute heart failure. Geriatr Gerontol Int. 2018;18:554-60.

32. Franch-Nadal J, Mata-Cases M, Vinagre I, et al. Differences in the cardiometabolic control in type 2 diabetes according to gender and the presence of cardiovascular disease: results from the eControl study. Int J Endocrinol. 2014;2014:131709.

33. Gamez JM, Masmiquel L, Ripoll T, Barrios V, Anguita M. Diabetes mellitus and cardiovascular clinical characteristics of Spanish women with stable ischaemic heart disease: data from the SIRENA study. Diabetes Res Clin Pract. 2017;123:82-6.

34. González-Juanatey JR, Alegría Ezquerra E, Gomis Barberá R, et al. Disfunción eréctil como marcador de vasculopatía en la diabetes mellitus tipo 2 en España. Estudio DIVA Med Clínica. 2009;132: 291-7.

35. Gual M, Formiga F, Ariza-Sole A, et al. Diabetes mellitus, frailty and prognosis in very elderly patients with acute coronary syndromes. Aging Clin Exp Res. 2019;31:1635-43.

36. Jimenez-Trujillo I, Gonzalez-Pascual M, JimenezGarcia R, et al. Type 2 diabetes mellitus and thoracic aortic aneurysm and dissection: an observational population-based study in Spain from 2001 to 2012. Med Baltim. 2016;95:e3618.

37. Jurado J, Ybarra J, Solanas P, et al. Prevalence of cardiovascular disease and risk factors in a type 2 diabetic population of the North Catalonia diabetes study. J Am Acad Nurse Pract. 2009;21:140-8.

38. López-de-Andres A, Jimenez-Trujillo I, JimenezGarcia R, et al. National trends in incidence and outcomes of abdominal aortic aneurysm among elderly type 2 diabetic and non-diabetic patients in Spain (2003-2012). Cardiovasc Diabetol. 2015;14: 48.

39. López-de-Andres A, Jimenez-Garcia R, HernandezBarrera V, et al. National trends over one decade in hospitalization for acute myocardial infarction among Spanish adults with type 2 diabetes: cumulative incidence, outcomes and use of percutaneous coronary intervention. PLoS ONE. 2014;9:e85697.

40. López-de-Andres A, Hernandez-Barrera V, CarrascoGarrido P, Esteban-Hernandez J, Gil-de-Miguel A, Jimenez-Garcia R. Trends of hospitalizations, fatality rate and costs for acute myocardial infarction 
among Spanish diabetic adults, 2001-2006. BMC Health Serv Res. 2010;10:59.

41. Navarro-Perez J, Orozco-Beltran D, Gil-Guillen V, et al. Mortality and cardiovascular disease burden of uncontrolled diabetes in a registry-based cohort: the ESCARVAL-risk study. BMC Cardiovasc Disord. 2018;18:180.

42. Mata-Cases M, Franch-Nadal J, Real J, Cedenilla M, Mauricio D. Prevalence and coprevalence of chronic comorbid conditions in patients with type 2 diabetes in Catalonia: a population-based crosssectional study. BMJ Open. 2019;9:e031281.

43. Mata-Cases M, Casajuana M, Franch-Nadal J, et al. Direct medical costs attributable to type 2 diabetes mellitus: a population-based study in Catalonia. Spain. Eur J Health Econ. 2016;17:1001-10.

44. Mata-Cases M, De Prado-Lacueva C, Salido-Valencia $\mathrm{V}$, et al. Incidence of complications and mortality in a type 2 diabetes patient cohort study followed up from diagnosis in a primary healthcare centre. Int J Clin Pract. 2011;65:299-307.

45. Mata-Cases M, Fernández-Bertolín E, García-Durán M, Cos-Claramunt X, Pareja-Rossell C, Pujol-Ribera E. Prevalencia de enfermedad cardiovascular en personas recien diagnosticadas de diabetes mellitus tipo 2. Gac Sanit. 2009;23:133-8.

46. Mostaza JM, Manzano L, Suarez C, et al. Different prognostic value of silent peripheral artery disease in type 2 diabetic and non-diabetic subjects with stable cardiovascular disease. Atherosclerosis. 2011;214:191-5.

47. Mundet X, Cano F, Mata-Cases M, et al. Trends in chronic complications of type 2 diabetic patients from Spanish primary health care centres (GEDAPS study): ten year-implementation of St. Vincent recommendations. Prim Care Diabetes. 2012;6: 11-8.

48. Muñoz-Rivas N, Jiménez-García R, Méndez-Bailón $\mathrm{M}$, et al. Type 2 diabetes increases the risk of hospital admission for heart failure and reduces the risk of in hospital mortality in Spain (2001-2015). Eur J Intern Med. 2019;59:53-9.

49. Muñoz-Rivas N, Mendez-Bailon M, Hernandez-Barrera $\mathrm{V}$, et al. Type 2 diabetes and hemorrhagic stroke: a population-based study in Spain from 2003 to 2012. J Stroke Cerebrovasc Dis. 2016;25: 1431-43.

50. Muñoz-Rivas N, Mendez-Bailon M, Hernandez-Barrera $\mathrm{V}$, et al. Time trends in ischemic stroke among type 2 diabetic and non-diabetic patients: analysis of the Spanish National Hospital discharge data (2003-2012). PLoS ONE. 2015;10:e0145535.
51. Orozco-Beltran D, Gil-Guillen VF, Redon J, et al. Lipid profile, cardiovascular disease and mortality in a Mediterranean high-risk population: the ESCARVAL-RISK study. PLOS ONE. 2017;12: e0186196.

52. Ortega E, Amor AJ, Rojo-Martinez G, Castell C, Gimenez M, Conget I. Cardiovascular disease in patients with type 1 and type 2 diabetes in Spain. Med Clin Barc. 2015;145:233-8.

53. Palanca A, Castelblanco E, Betriu A, et al. Subclinical atherosclerosis burden predicts cardiovascular events in individuals with diabetes and chronic kidney disease. Cardiovasc Diabetol. 2019;18:93.

54. Peña-Longobardo LM, Rodriguez-Sanchez B, MataCases M, Rodriguez-Manas L, Capel M, Oliva-Moreno J. Is quality of life different between diabetic and non-diabetic people? The importance of cardiovascular risks. PLoS ONE. 2017;12:e0189505.

55. Pérez A, Mediavilla JJ, Minambres I, Gonzalez-Segura D. Glycemic control in patients with type 2 diabetes mellitus in Spain. Rev Clin Esp. 2014;214: 429-36.

56. Piniés JA, Gonzalez-Carril F, Arteagoitia JM, et al. Development of a prediction model for fatal and non-fatal coronary heart disease and cardiovascular disease in patients with newly diagnosed type 2 diabetes mellitus: the Basque Country Prospective Complications and Mortality Study risk engine (BASCORE). Diabetologia. 2014;57:2324-33.

57. Ramírez-Prado D, Palazon-Bru A, Folgado-de-la Rosa DM, Carbonell-Torregrosa MA, Martinez-Diaz AM, Gil-Guillen VF. Predictive models for all-cause and cardiovascular mortality in type 2 diabetic inpatients. A cohort study. Int J Clin Pract. 2015;69: 474-84.

58. Ramos R, Comas-Cufi M, Marti-Lluch R, et al. Statins for primary prevention of cardiovascular events and mortality in old and very old adults with and without type 2 diabetes: retrospective cohort study. BMJ. 2018;362:k3359.

59. Rodríguez-Poncelas A, Coll-De Tuero G, Turro-Garriga $\mathrm{O}$, Barrot-de la Puente J, Franch-Nadal J, Mundet-Tuduri X. Impact of chronic kidney disease on the prevalence of cardiovascular disease in patients with type 2 diabetes in Spain: PERCEDIME2 study. BMC Nephrol. 2014;15:150.

60. Salinero-Fort MA, San Andres-Rebollo FJ, de BurgosLunar C, et al. Cardiovascular and all-cause mortality in patients with type 2 diabetes mellitus in the MADIABETES cohort study: association with chronic kidney disease. J Diabetes Complicat. 2016;30:227-36. 
61. Salinero-Fort MA, Mostaza-Prieto JM, Lahoz-Rallo C, Vicente Diez JI, Cardenas-Valladolid J. Population-based cross-sectional study of 11645 Spanish nonagenarians with type 2 diabetes mellitus: cardiovascular profile, cardiovascular preventive therapies, achievement goals and sex differences. BMJ Open. 2019;9:e030344.

62. Sancho-Mestre C, Vivas-Consuelo D, Alvis-Estrada L, Romero M, Uso-Talamantes R, Caballer-Tarazona V. Pharmaceutical cost and multimorbidity with type 2 diabetes mellitus using electronic health record data. BMC Health Serv Res. 2016;16:394.

63. Sicras-Mainar A, Navarro-Artieda R. Use of metformin and vildagliptin for treatment of type 2 diabetes in the elderly. Drug Dev Ther. 2014;8: 811-8.

64. Sicras-Mainar A, Navarro-Artieda R, Ibanez-Nolla J. Clinical and economic characteristics associated with type 2 diabetes. Rev Clin Esp Barc. 2014;214: 121-30.

65. Vinagre I, Mata-Cases M, Hermosilla E, et al. Control of glycemia and cardiovascular risk factors in patients with type 2 diabetes in primary care in Catalonia (Spain). Diabetes Care. 2012;35:774-9.

66. Zapatero-Gaviria A, Gómez-Huelgas R, Canora-Lebrato J, et al. Analysis of hospitalizations by cardiovascular disease in the population with diabetes in Spain. Rev Clin Esp. 2019;219:124-9.

67. Arrieta F, Iglesias P, Pedro-Botet J, et al. Diabetes mellitus and cardiovascular risk: update of the recommendations of the Diabetes and Cardiovascular Disease working group of the Spanish Diabetes Society (SED, 2018). Clin Investig Arter. 2018;30: 137-53.

68. Fox CS, Golden SH, Anderson C, et al. Update on prevention of cardiovascular disease in adults with type 2 diabetes mellitus in light of recent evidence: a scientific statement from the American Heart Association and the American Diabetes Association. Diabetes Care. 2015;38:1777-803.

69. Rawshani A, Franzen S, Sattar N, et al. Risk factors, mortality, and cardiovascular outcomes in patients with type 2 diabetes. N Engl J Med. 2018;379: 633-44.

70. Newman JD, Schwartzbard AZ, Weintraub HS, Goldberg IJ, Berger JS. Primary prevention of cardiovascular disease in diabetes mellitus. J Am Coll Cardiol. 2017;70:883-93.

71. Das SR, Everett BM, Birtcher KK, et al. 2020 Expert Consensus Decision Pathway on novel therapies for cardiovascular risk reduction in patients with type 2 diabetes: a report of the American College of
Cardiology Solution Set Oversight Committee. J Am Coll Cardiol. 2020;76:1117-45.

72. van der Heijden AA, Van't Riet E, Bot SD, et al. Risk of a recurrent cardiovascular event in individuals with type 2 diabetes or intermediate hyperglycemia: the Hoorn study. Diabetes Care. 2013;36: 3498-502.

73. Raghavan S, Vassy JL, Ho YL, et al. Diabetes mellitus-related all-cause and cardiovascular mortality in a national cohort of adults. J Am Heart Assoc. 2019;8:e011295.

74. Gaede P, Oellgaard J, Carstensen B, et al. Years of life gained by multifactorial intervention in patients with type 2 diabetes mellitus and microalbuminuria: 21 years follow-up on the Steno2 randomised trial. Diabetologia. 2016;59: 2298-307.

75. Rao Kondapally Seshasai S, Kaptoge S, Thompson A, et al. Diabetes mellitus, fasting glucose, and risk of cause-specific death. N Engl J Med. 2011;364: 829-41.

76. Gregg EW, Sattar N, Ali MK. The changing face of diabetes complications. Lancet Diabetes Endocrinol. 2016;4:537-47.

77. Gaeta M, Campanella F, Gentile L, et al. European cardiovascular mortality over the last three decades: evaluation of time trends, forecasts for 2016. Ann Ig. 2017;29:206-17.

78. Dauriz M, Mantovani A, Bonapace S, et al. Prognostic impact of diabetes on long-term survival outcomes in patients with heart failure: a metaanalysis. Diabetes Care. 2017;40:1597-605.

79. Li R, Bilik D, Brown MB, et al. Medical costs associated with type 2 diabetes complications and comorbidities. Am J Manag Care. 2013;19:421-30.

80. Lopez-Bastida J, Boronat M, Moreno JO, Schurer W. Costs, outcomes and challenges for diabetes care in Spain. Glob Health. 2013;9:17.

81. Zozaya N, Villoro R, Hidalgo Á, Oliva J, Rubio M, García S. Estudios de coste de la diabetes tipo 2: una revisión de la literatura. Agencia Eval Tecnol Sanit Inst Salud Carlos III Minist Econ Compet. 2015. http://gesdoc.isciii.es/gesdoccontroller?action= download\&id=26/05/2015-28ff538b32. Accessed 15 Jan 2020.

82. Jodar E, Artola S, Garcia-Moll X, Uría E, LópezMartínez N, Palomino R, et al. Incidence and costs of cardiovascular events in Spanish patients with type 2 diabetes mellitus: a comparison with general population, 2015. BMJ Open Diabetes Res Care. 2020;8:e001130. 
83. Gómez-Martínez L, Orozco-Beltrán D, Quesada JA, et al. Trends in premature mortality due to heart failure by Autonomous Community in Spain: 1999 to 2013. Rev Esp Cardiol Engl Ed. 2018;71:531-7.
84. Orozco-Beltran D, Sanchez E, Garrido A, Quesada JA, Carratala-Munuera MC, Gil-Guillen VF. Trends in mortality from diabetes mellitus in Spain: 1998-2013. Rev Esp Cardiol Engl Ed. 2017;70:433-43. 